\title{
Block copolymers of "PE-like" poly(pentadecalactone) and poly(L-lactide): synthesis, properties, and compatibilization of polyethylene/poly(L-lactide) blends
}

\author{
Citation for published version (APA): \\ Pepels, M. P. F., Hofman, W. P., Kleijnen, R. G., Spoelstra, A. B., Koning, C. E., Goossens, H., \& Duchateau, R. \\ (2015). Block copolymers of "PE-like" poly(pentadecalactone) and poly(L-lactide): synthesis, properties, and \\ compatibilization of polyethylene/poly(L-lactide) blends. Macromolecules, 48(19), 6909-6921. \\ https://doi.org/10.1021/acs.macromol.5b01620
}

DOI:

10.1021/acs.macromol.5b01620

Document status and date:

Published: 13/10/2015

\section{Document Version:}

Publisher's PDF, also known as Version of Record (includes final page, issue and volume numbers)

\section{Please check the document version of this publication:}

- A submitted manuscript is the version of the article upon submission and before peer-review. There can be important differences between the submitted version and the official published version of record. People interested in the research are advised to contact the author for the final version of the publication, or visit the DOI to the publisher's website.

- The final author version and the galley proof are versions of the publication after peer review.

- The final published version features the final layout of the paper including the volume, issue and page numbers.

Link to publication

\footnotetext{
General rights

- You may freely distribute the URL identifying the publication in the public portal. follow below link for the End User Agreement:

www.tue.nl/taverne

\section{Take down policy}

If you believe that this document breaches copyright please contact us at:

openaccess@tue.nl

providing details and we will investigate your claim.
}

Copyright and moral rights for the publications made accessible in the public portal are retained by the authors and/or other copyright owners and it is a condition of accessing publications that users recognise and abide by the legal requirements associated with these rights.

- Users may download and print one copy of any publication from the public portal for the purpose of private study or research.

- You may not further distribute the material or use it for any profit-making activity or commercial gain

If the publication is distributed under the terms of Article $25 \mathrm{fa}$ of the Dutch Copyright Act, indicated by the "Taverne" license above, please 


\title{
Block Copolymers of "PE-Like" Poly(pentadecalactone) and Poly(L-lactide): Synthesis, Properties, and Compatibilization of Polyethylene/Poly(L-lactide) Blends
}

\author{
Mark P. F. Pepels, ${ }^{\dagger}$ Wilma P. Hofman, ${ }^{\dagger}$ Rob Kleijnen, ${ }^{\dagger}$ Anne B. Spoelstra, ${ }^{\dagger}$ Cor E. Koning, \\ Han Goossens, ${ }^{\dagger}$ and Rob Duchateau* ${ }^{*} \dagger, \S$ \\ ${ }^{\dagger}$ Laboratory of Polymer Materials, Department of Chemical Engineering and Chemistry, Eindhoven University of Technology, P.O. \\ Box 513, $5600 \mathrm{MB}$ Eindhoven, The Netherlands \\ ${ }^{\S}$ SABIC T\&I, STC-Geleen, SABIC Europe B.V., Urmonderbaan 22, 6160 AH Geleen, The Netherlands
}

Supporting Information

\begin{abstract}
Block copolymers consisting of a polyethylene block and a polar polymer block are interesting structures for the compatibilization of polyethylene/polar polymer blends or polyethylene-based composites. Since the synthesis of polyethylenebased block copolymers is an elaborate process, diblock copolymers consisting of "polyethylene-like" poly(pentadecalactone) (PPDL) and poly(L-lactide) (PLLA) were synthesized using a one-pot, sequential-feed ring-opening polymerization of pentadecalactone (PDL) and L-lactide (LLA). The peculiar activity of the used aluminum salen catalysts yielded a block copolymer consisting of two blocks with both a high dispersity, as a result of intrablock transesterification. Interestingly, interblock transesterification was effectively suppressed. The

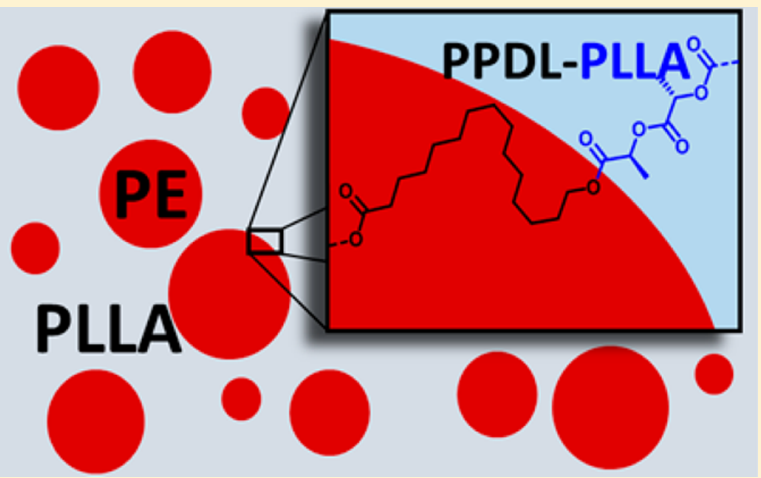
obtained poly(PDL-block-LLA) of various block lengths showed coincidental crystallization of the two blocks with an associated microphase-separated morphology, in which PLLA spheres with a high dispersity are distributed within the PPDL matrix. The complex morphologies is believed to arise from the presence of a whole range of block sizes as a consequence of the large dispersity of both blocks. The application of these block copolymers as compatibilizers for high density polyethylene (HDPE)/PLLA blends led to a clear change in blend morphology and a steep decrease in particle size of the dispersed phase. Furthermore, addition of the block copolymers to blends of linear low density polyethylene (LLDPE) and PLLA led to a significant increase in adhesion between the two phases. For both HDPE/PLLA and LLDPE/PLLA blends, the compatibilization efficiency of the poly(PDL-block-LLA) increased when the length of the PPDL block was increased. The presented results clearly show that PPDL can function as a substituent for various types of polyethylene, which opens up a new method for compatibilizing polyethylene with polar polymers using easy attainable "PE-like" block copolymers.
\end{abstract}

\section{INTRODUCTION}

Introducing polar components in a controlled manner in polyolefin architectures is a frequently applied method to add extra functionality to the properties of these polymers, e.g., printability and barrier properties, ${ }^{1}$ or to effectively compatibilize polyolefin/polar polymer blends and to enhance the interaction with fillers. However, widespread application of block copolymers of polyolefins with polar counits is impeded by the rather tedious procedures required to make them, involving multiple step reactions, functionalization of intermediates and incompatibility of the catalyst systems required for the various reactions. ${ }^{2-6}$

Recently, it was found that polymacrolactones and related polyesters having a large methylene/ester ratio have properties resembling polyethylene. ${ }^{7,8}$ Hence, these polymers could function as polyethylene mimics and block copolymers based on such polyesters with polyethylene-like properties can be prepared easily by the sequential ring-opening copolymerization (ROP) of macrolactones and smaller polar lactones. ${ }^{9-11}$ Copolymerization of macrolactones and small-ring (strained) lactones has been reported by several authors. ${ }^{9-14}$ Even though control in these types of polymerization is generally difficult due to the low ring strain of the macrolactone and the associated entropic nature of the polymerization, ${ }^{15}$ stable block copolymer architectures could be synthesized using a sequential feed polymerization approach, ${ }^{14,16}$ or even by a single-feed polymerization. $^{11}$

Received: July 20, 2015

Revised: September 6, 2015

Published: September 24, 2015 
Scheme 1. Ring-Opening Polymerization of PDL and LLA Catalyzed by Aluminum Salen Complexes $\mathbf{1}^{\prime}$ and $2^{\prime}$

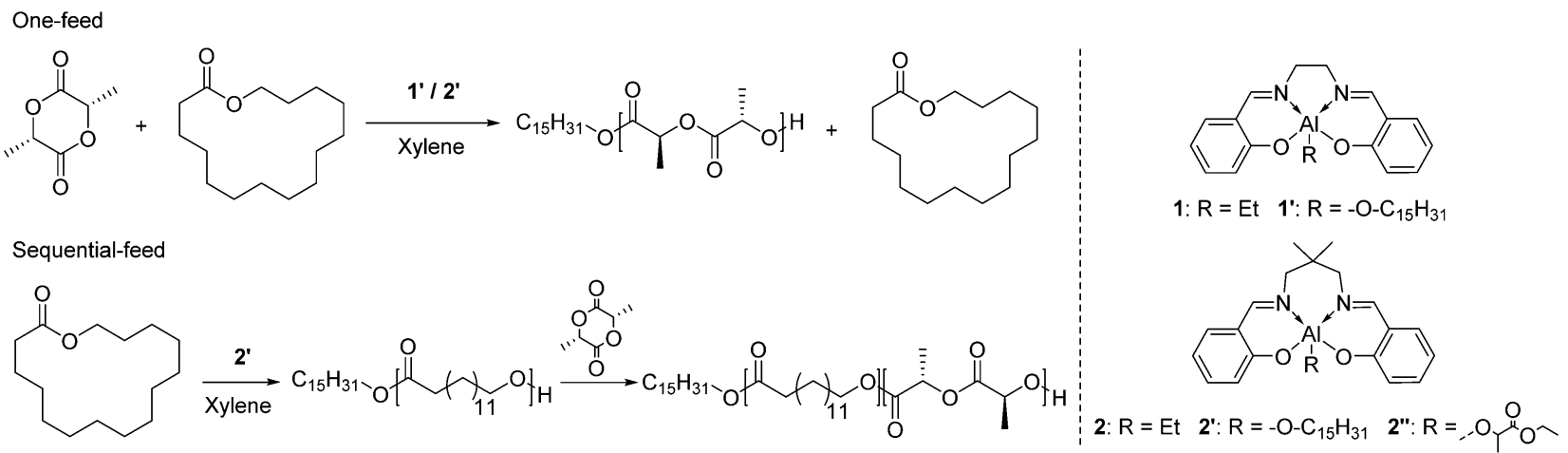

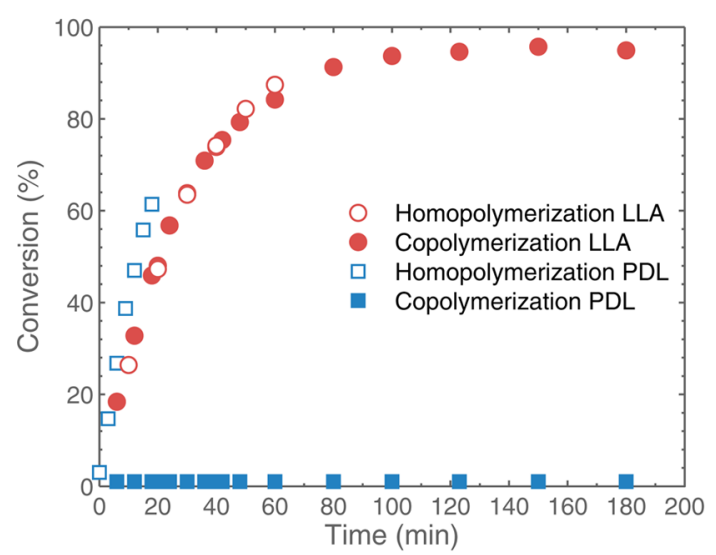

(a)

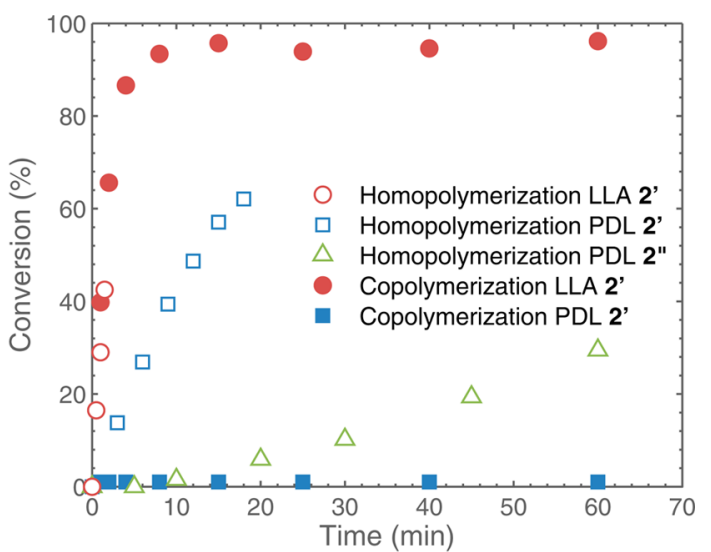

(b)

Figure 1. Homopolymerization and copolymerization of PDL and LLA using $\mathbf{1}^{\prime}$ (a) and $\mathbf{2}^{\prime} / \mathbf{2}^{\prime \prime}$ (b) (see Scheme 1).

L-Lactide (LLA) is a polar lactone that has been studied extensively over the last decades and the associated polymer, poly(L-lactide) (PLLA), is considered to be one of the most economically viable biobased polymers. ${ }^{17}$ Block copolymers of polyethylene (PE) and PLLA have been reported to exhibit strong segregation of the two blocks due to the incompatibility of the two polymers. ${ }^{5,6,18-20}$ Furthermore, these block copolymers showed high compatibilization efficiency in PE/ PLLA blends. ${ }^{18,21}$ The block copolymer synthesis, however, required several steps. For the sake of feasibility, it would therefore be desired to replace the PE block by a "PE-like" polyester block, in which case the compatibilizer would consist of easy to synthesize $A B$ diblock or $A B A$ triblock copolyesters. Poly(pentadecalactone) (PPDL) is one of the polymacrolactones that has been shown to exhibit PE-like properties as a result of the large amount of methylene units compared to the ester units in the backbone. ${ }^{7,22}$ Decreasing the ratio of methylene versus ester groups results in a gradual decrease of the melting temperature and a gradual increase of the polarity of the material. ${ }^{23,24}$ Even though "PE-like" polyesters such as PPDL are not fully miscible with PE, the similarity of the crystal lattice dimension of both polymers leads to epitaxial crystallization of PPDL from the PE lamellae, which strongly increases the adhesion between the two phases. ${ }^{25}$

Recently, Todd et al. reported the synthesis of PPDL-blockPLLA copolymers using a sequential feed polymerization of PDL and LLA using organic catalysts, in which the consecutive polymerization steps were performed at different temperatures in order to avoid transesterification. These copolymers were shown to be effective compatibilizers for PLLA/poly $(\omega$ hydroxytetradecanoate) blends, which results from the molecular similarity of PPDL and poly $(\omega$-hydroxytetradecanoate) (methylene/ester group ratio $=14$ and 13, respectively). ${ }^{26}$ In other work, the compatibility of PLLA $/ \operatorname{poly}(\omega$ hydroxytetradecanoate) blends was enhanced by the in situ generation of block copolymers through transesterification reactions between both polymers during reactive extrusion. ${ }^{27}$ However, the application of polymacrolactones and related polyesters as PE-mimicking blocks in corresponding block copolymer compatibilizers has never been attempted so far.

Here we describe the synthesis of amphiphilic PPDL-blockPLLA copolymers by a one-pot, sequential-feed ring-opening polymerization (ROP) of PDL and LLA using a single catalytic system, avoiding the isolation of reaction intermediates. Furthermore, the crystallization behavior and morphology of block copolymers with various block lengths is described. Finally, in order to investigate the "PE-like" character of PPDL, the compatibilization potential of these block copolymers for PE/PLLA blends is being evaluated.

\section{RESULTS AND DISCUSSION}

Copolymerization and Characterization. In a previous study we showed the high activity of aluminum salen catalysts (Scheme $1, \mathbf{1}^{\prime}$ and $\mathbf{2}^{\prime}$ ) toward the ROP of both macrolactones and small ring lactones. ${ }^{9}$ Because of the rapid transesterification of copolymers of PDL and $\varepsilon$-caprolactone (CL), which was ascribed to the identical nature of the polymerization of 
Table 1. Copolymerization of PDL and LLA Using a Sequential Feed Strategy ${ }^{a}$

\begin{tabular}{|c|c|c|c|c|c|c|c|c|c|c|}
\hline \multirow[b]{2}{*}{ entry } & \multicolumn{2}{|c|}{$[\mathrm{M}]_{0} /\left[2^{\prime}\right]$} & \multicolumn{2}{|c|}{ time $(\min )$} & \multicolumn{2}{|c|}{ Conversion $(\%)^{b}$} & \multicolumn{3}{|c|}{ molecular weight $\left(\mathrm{kg} \cdot \mathrm{mol}^{-1}\right)^{c}$} & \multirow[b]{2}{*}{$\bigoplus_{\mathrm{M}}$} \\
\hline & PDL & LLA & PDL & LLA & PDL & LLA & $M_{\mathrm{n}, \mathrm{calc}}^{d}$ & $M_{\mathrm{n}}$ & $M_{\mathrm{w}}$ & \\
\hline PPL1 & 50 & 0 & 35 & - & 97.8 & - & 12.0 & 27.6 & 50.5 & 1.8 \\
\hline PPL2 & 50 & 62 & 35 & 10 & 96.4 & 98.1 & 20.6 & 41.2 & 69.7 & 1.7 \\
\hline PPL3 & 50 & 113 & 35 & 12 & 96.6 & 97.8 & 27.8 & 57.4 & 95.5 & 1.7 \\
\hline PPL4 & 50 & 208 & 35 & 18 & 94.3 & 96.7 & 40.5 & 84.4 & 132 & 1.6 \\
\hline PPL5 & 47 & 481 & 35 & 38 & 94.7 & 92.4 & 75.0 & 144 & 210 & 1.5 \\
\hline PPL6 & 47 & 1067 & 35 & 70 & 94.7 & 48.6 & 85.7 & 115 & 132 & 1.2 \\
\hline OPL1 & 20 & 0 & 30 & - & 98.3 & - & 5.0 & 7.3 & 20.2 & 2.8 \\
\hline OPL2 & 0 & 20 & - & 20 & - & 99.2 & 3.1 & 4.2 & 10.2 & 2.4 \\
\hline OPL3 & 10 & 13 & 30 & 10 & 96.9 & 98.9 & 4.4 & 8.3 & 14.6 & 1.8 \\
\hline
\end{tabular}

${ }^{a} \mathrm{~T}=100{ }^{\circ} \mathrm{C},[\mathrm{I}]_{0}=23 \mathrm{mmol}$, solvent $=p$-xylene. ${ }^{b}$ Conversion is determined by ${ }^{1} \mathrm{H}$ NMR spectroscopy from the $\alpha$-methylene resonances. ${ }^{c}$ Measured using SEC (chloroform) relative to PS standards. ${ }^{d}$ calculated using $\left([\mathrm{PDL}]_{0} /[\mathrm{Cat}] \times\right.$ convn PDL $\left.\times 240.4\right)+\left([\mathrm{LLA}]_{0} /[\mathrm{Cat}] \times\right.$ convn LLA $\times 144.1)+228.4$.

strainless rings (PDL) and transesterification of linear polymer chains, it proved very difficult to form block copolymers by sequential monomer feed using aluminum salen catalysts. ${ }^{9}$

To investigate the copolymerization behavior for PDL and LLA, the single-feed-reaction of both monomers $([\mathrm{PDL}]=0.50$ $\mathrm{M},[\mathrm{LLA}]=0.50 \mathrm{M})$ in $p$-xylene was studied at $100{ }^{\circ} \mathrm{C}$ using both [Salen]AlOPD $\left(\mathbf{1}^{\prime}, 0.01 \mathrm{M}\right)$ and [SalDMPN]AlOPD (2', $0.01 \mathrm{M})$. Catalysts $\mathbf{1}^{\prime}$ and $\mathbf{2}^{\prime}$ were prepared in situ by reacting [Salen]AlEt (1) and [SalDMPN]AlEt (2) with 1-pentadecanol $(\mathrm{PDOH})$. During the copolymerization reactions, the conversion over time of LLA was similar to the profile obtained from the independent homopolymerizations using both catalysts $\mathbf{1}^{\prime}$ and $\mathbf{2}^{\prime}$ (Figure 1). However, in contrast to the PDL homopolymerization, no conversion of PDL was observed regardless which of the two catalysts was used. Even after prolonged reaction times of up to $90 \mathrm{~h}$ at elevated temperatures $\left(130^{\circ} \mathrm{C}\right)$ and complete conversion of the LLA, no significant conversion of PDL could be observed (Supporting Information, Table S1). Hence, it appears that once an LLA monomer has inserted into the growing polymer chain and is attached to the catalytically active site, insertion of a PDL unit is inhibited.

In an attempt to investigate this peculiar catalytic behavior of the aluminum salen catalyst further, $\mathbf{2}$ was treated with one equivalent of $L$-ethyllactate (LEL) to in situ form the aluminum lactyl, [SalDMPN]Al-OCH(Me) $\mathrm{CO}_{2} \mathrm{Et}$ (2"), mimicking a catalyst with a growing polylactide chain. Polymerization of $\mathrm{PDL}([\mathrm{PDL}]=1.00 \mathrm{M})$ using this catalyst $\left(\left[\mathbf{2}^{\prime \prime}\right]=0.01 \mathrm{M}\right)$ shows that the initiation is significantly retarded (Figure $1 \mathrm{~b}$ ) and even after $60 \mathrm{~min}$ the polymerization rate is still increasing. The fact that PDL polymerization is not inhibited completely, as has been observed during the attempted copolymerization of PDL with LLA, is likely the result of transesterification of two aluminum-LEL species, resulting in two different initiating species, i.e. the aluminum ethyllactidyl ([SalDMPN]Al$\left.\mathrm{OCH}(\mathrm{Me}) \mathrm{CO}_{2} \mathrm{CH}(\mathrm{Me}) \mathrm{CO}_{2} \mathrm{Et}\right)$ and the sterically unhindered aluminum ethoxide ([SalDMPN] Al-OEt). The latter most probably can polymerize PDL and its gradual formation by transesterification would therefore explain the progressive increase of the PDL polymerization rate over time.

The hypothesis that the growing PLLA chain effectively blocks the active site for incorporation of PDL was further strengthened by the attempted polymerization of PDL ([PDL] $\left.=0.95 \mathrm{M},\left[\mathbf{2}^{\prime}\right]=0.01 \mathrm{M}\right)$ in the presence of a small amount of lactide $([\mathrm{LLA}]=0.1 \mathrm{M})$. This reaction resulted in only $3.8 \%$ conversion of PDL after $60 \mathrm{~min}$ reaction at $130{ }^{\circ} \mathrm{C}$, showing the extremely low tolerance of a growing PLLA chain toward incorporation of PDL. This is in agreement with results found for the copolymerization of LLA and CL using $\mathrm{Zn}$ and $\mathrm{Mg}$ complexes, where the chelating effect of the lactate unit was shown to be responsible for the inability of these complexes to facilitate copolymerization, while both monomers were efficiently homopolymerized. ${ }^{28,29}$

Since the PLLA growing chain showed no reactivity toward PDL and the ROP of a strainless cyclic ester such as PDL resembles the transesterification of a linear chain, it was assumed that a growing PLLA chain would not be able to undergo transesterification with a PPDL chain either. Therefore, in an attempt to produce a poly(PDL-block-LLA) copolymer, a sequential feed approach was applied in which first PDL was allowed to polymerize to $>94 \%$ monomer conversion using $\mathbf{2}^{\prime}\left([\mathrm{PDL}] /\left[\mathbf{2}^{\prime}\right]=50\right)$, after which varying amounts of lactide (as hot $p$-xylene solutions) were added. This resulted in high conversions for both monomers yielding diblock copolymers containing an average of 50 PDL units and 0 to 500 LLA units (Table 1, PPL1-PPL5).

For the reaction having the highest LLA to catalyst ratio (PPL6), the LLA conversion did not approach completion during the time frame of the experiment. A likely reason for this is the partial catalyst deactivation by small amounts of impurities in the monomer.

To be able to analyze the blockiness of the copolymers by NMR and MALDI-ToF-MS, next to high molecular weight polymers also a series of low molecular weight (co)oligomers was synthesized (OPL1-OPL3).

The molecular weights (determined by SEC) show a linear increase relative to the converted LLA (Figure 2). The experimental molecular weights are higher than the calculated ones, which is most likely caused by the fact that the SEC apparatus was calibrated using PS standards. ${ }^{30,31}$ Furthermore, increasing $[\mathrm{LLA}]_{0} /[\mathrm{Cat}]$ results in increasing impurity levels and subsequently more catalyst deactivation and a higher effective LLA/catalyst ratio. The dispersity of PPDL (PPL1) is around two, as is expected for the linear chain fraction of a polymer which is subjected to extensive transesterification reactions. For the corresponding block copolymers, slightly lower dispersities are obtained suggesting that transesterification of the PLLA block is limited or absent during the time frame of the experiment.

The DSC thermograms of all copolymers show two melting transitions (Figure 3 for PPL3), viz. one around $90{ }^{\circ} \mathrm{C}$, which corresponds to PPDL, and one around $165^{\circ} \mathrm{C}$, corresponding to PLLA. This is the typical behavior for block copolymers in 


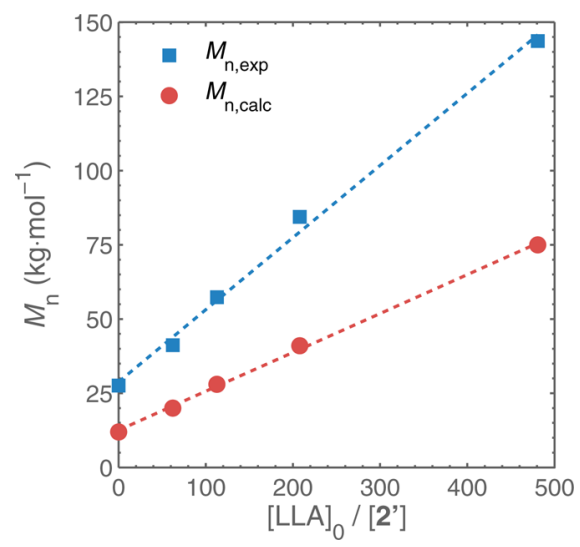

Figure 2. Molecular weight of the polymers formed for the polymerizations using $[\mathrm{PDL}]_{0} /[\mathrm{I}]_{0}=50$ and various ratios of LLA (PPL1-PPL5 of Table 1).

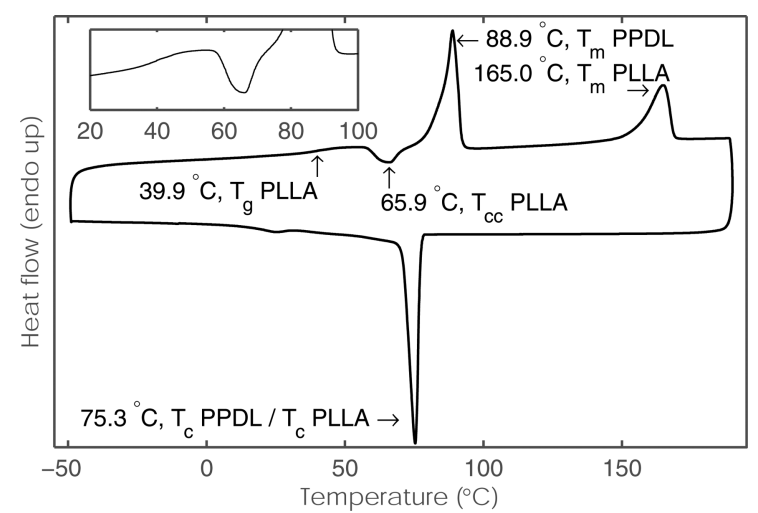

Figure 3. DSC thermogram of the first cooling and second heating run for PPL3.

which both blocks can crystallize individually in microphaseseparated domains. ${ }^{32}$ The $T_{\mathrm{g}}$ of PLLA, taken as the inflection point of the step in the heat flow, is observed at $39.9{ }^{\circ} \mathrm{C}$ (see inset Figure 3). Furthermore, the PLLA-block shows a cold crystallization peak above its $T_{\mathrm{g}}$ around $66{ }^{\circ} \mathrm{C}$ (Figure 3). It should be noted that this peak distorts the shape of the PPDL melting peak due to which it is not possible to determine the melting enthalpy for the PPDL-block. The thermogram shows only one clear crystallization peak, corresponding to the crystallization of PPDL. However, it cannot be excluded that PLLA partly crystallizes in this regime. For now the most important fact is the observation of two melting endotherms, corresponding to a phase-separated block copolymer structure, which confirms the assumption that a PLLA chain does not transesterify with a PPDL chain. Further on, a more detailed analysis of the thermal properties is described.

In order to elaborate more on the molecular structure of the copolymers, ${ }^{13} \mathrm{C}$ NMR spectroscopy was applied. For PPL1 PPL6, the observed signals only correspond to homopolymer diads (PDL-PDL at $64.53 \mathrm{ppm}$ and LLA-LLA at $69.14 \mathrm{ppm}$ ), of which a typical example is shown in Figure 4a. The lack of signals originating from end-groups or linkages between PDL and LLA is caused by their relative low concentration. However, analysis and peak assignment of the co-oligomer (OPL3, Figure $4 \mathrm{~b}$ ) using a combination of ${ }^{13} \mathrm{C}$ NMR and 2D ${ }^{1} \mathrm{H}-{ }^{13} \mathrm{C}$ HSQC NMR revealed the PDL-LLA diads at 69.44, 69.23 ( $\mathrm{PPDL}-\mathrm{C}(=\mathrm{O}) \mathrm{C}(\mathrm{H}) \mathrm{MeOC}(=\mathrm{O}) \mathrm{C}(\mathrm{H}) \mathrm{MeO}-\mathrm{PLLA})$ and $65.83 \mathrm{ppm}$ (PPDL-C $\left.(=\mathrm{O})\left(\mathrm{CH}_{2}\right)_{13} \mathrm{CH}_{2} \mathrm{O}-\mathrm{PLLA}\right)$. The signal at $66.84 \mathrm{ppm}$ was assigned to the PLLA end-group. ${ }^{33}$ The presence of the PDL-LLA diads in ${ }^{13} \mathrm{C} \mathrm{NMR,} \mathrm{in}$ combination with the increase in molecular weight obtained from SEC and the two individual melting peaks for both blocks observed in DSC is convincing evidence for the formation of poly(PDL-block-LLA) copolymers.

As a final tool to elucidate the molecular structure and the associated reaction mechanism, MALDI-ToF-MS analysis was performed on the copolymers. The fact that 3 PDL units and 5 LLA units result in the same mass somewhat frustrated the analysis. For the high molecular weight copolymers only distributions were found corresponding to the cyclic homopolymers of PPDL and PLLA (Figure 5a). As stated before, aluminum salen catalysts are good transesterification catalysts, which, in accordance to the Jacobson-Stockmayer theory, results in cyclic chains in the low molecular weight regime. These cyclic oligomers are present in excess compared to their low molecular weight linear counterparts. ${ }^{15,34}$

However, it is peculiar that this transesterification does lead to cyclic PPDL and PLLA, but not to cyclic copolymers consisting of a combination of PDL and LLA. For example, a peak is present at $1706.1 \mathrm{~g} \cdot \mathrm{mol}^{-1}$, which corresponds to cyclic $(\mathrm{PDL})_{7}+\mathrm{Na}^{+}$, while there is no peak present at $1706.1+$ $x 72.06 \mathrm{~g} \cdot \mathrm{mol}^{-1}$ ( $x$ lactidyl units). However, the presence of a repeating unit of $72 \mathrm{~g} \cdot \mathrm{mol}^{-1}$ having a similar intensity as the repeating unit of $144 \mathrm{~g} \cdot \mathrm{mol}^{-1}$, does suggest rapid transesterification within PLLA segments. This is a striking observation, since the ester group of a PDL repeating unit appears less sterically hindered than a PLLA repeating unit and would therefore be expected to undergo faster transesterification with a metal center connected to a growing PLLA chain than PLLA itself. The exact reason for this effect currently remains unknown. The MALDI-ToF-MS spectrum of OPL3 shows several extra signals compared to PPL5, corresponding to distributions having a combination of a $\mathrm{PDO}-$ and an $-\mathrm{H}$

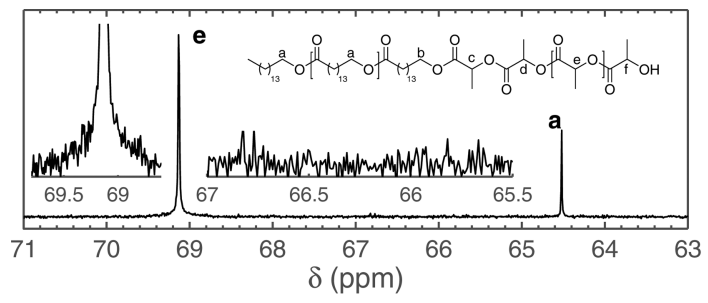

(a)

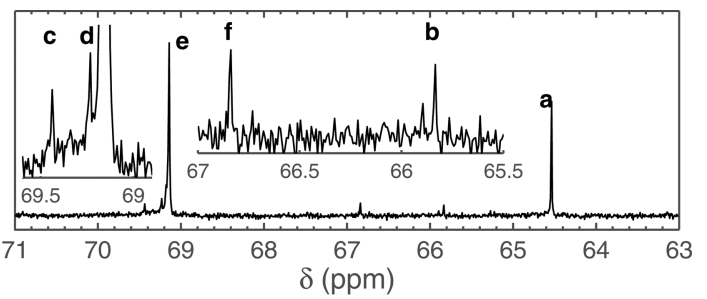

(b)

Figure 4. ${ }^{13} \mathrm{C}$ NMR samples of PPL3 (a) and OPL3 (b). 


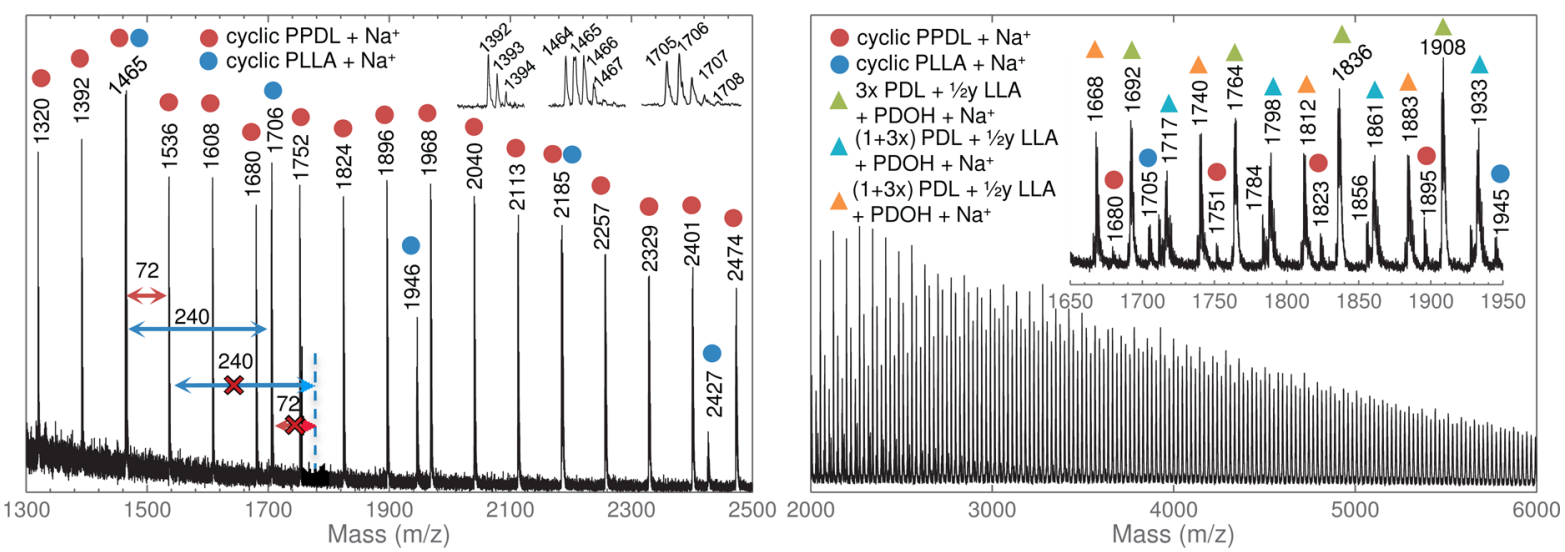

(a)

(b)

Figure 5. MALDI-ToF MS spectra of (a) PPL5 and (b) OPL3.

Table 2. Composition of Poly(PDL-block-LLA) Copolymers Produced on a Large Scale ${ }^{a}$

\begin{tabular}{|c|c|c|c|c|c|c|c|c|c|c|c|c|}
\hline \multirow[b]{2}{*}{ entry } & \multicolumn{2}{|c|}{$[\mathrm{M}]_{0} /\left[2^{\prime}\right]$} & \multicolumn{2}{|c|}{ time $(\mathrm{min})$} & \multicolumn{2}{|c|}{$\operatorname{convn}(\%)^{b}$} & \multicolumn{2}{|c|}{ mass fraction } & \multicolumn{4}{|c|}{ molecular weight $\left(\mathrm{kg} \cdot \mathrm{mol}^{-1}\right)^{c}$} \\
\hline & PDL & LLA & PDL & LLA & PDL & LLA & PDL & LLA & $M_{\mathrm{n}, \mathrm{calc}}{ }^{d}$ & $M_{\mathrm{n}}$ & $M_{\mathrm{w}}$ & $\emptyset_{M}$ \\
\hline P0L100 & 0 & 335 & 0 & 60 & - & 98.6 & 0 & 1 & 47.8 & 69 & 118 & 1.7 \\
\hline P26L74 & 50 & 232 & 60 & 60 & 96.6 & 98.3 & 0.26 & 0.74 & 44.7 & 61 & 112 & 1.8 \\
\hline P52L48 & 100 & 151 & 120 & 60 & 96.1 & 97.6 & 0.52 & 0.48 & 44.6 & 60 & 103 & 1.7 \\
\hline P77L23 & 150 & 75 & 180 & 60 & 97.3 & 97.2 & 0.77 & 0.23 & 45.8 & 62 & 108 & 1.8 \\
\hline P100L0 & 199 & 0 & 240 & - & 96.7 & - & 1 & 0 & 46.5 & 81 & 134 & 1.7 \\
\hline
\end{tabular}

${ }^{a} \mathrm{~T}=100{ }^{\circ} \mathrm{C}, m_{\mathrm{PDL}}+m_{\mathrm{LLA}}=15 \mathrm{~g}$, solvent $=$ toluene. ${ }^{b}$ Conversion was determined by ${ }^{1} \mathrm{H}$ NMR spectroscopy from the $\alpha$-methylene resonances. ${ }^{c}$ Measured using SEC (chloroform) relative to PS standards. ${ }^{d}$ Calculated using $\left([\mathrm{PDL}]_{0} /[\mathrm{Cat}] \times\right.$ convn PDL $\left.\times 240.4\right)+\left([\mathrm{LLA}]_{0} /[\mathrm{Cat}] \times\right.$ convn LLA $\times 144.1)+228.4$.

Table 3. Thermal Properties of the PPDL, PLLA, and Poly(PDL-block-LLA) Copolymers Produced on a Large Scale

\begin{tabular}{|c|c|c|c|c|c|c|c|c|c|}
\hline & \multicolumn{2}{|c|}{ wt $\%$} & \multicolumn{2}{|c|}{ DMTA } & \multicolumn{5}{|c|}{ DSC } \\
\hline & PDL & LLA & $T_{\mathrm{g}, \mathrm{PPDL}}{ }^{a}\left({ }^{\circ} \mathrm{C}\right)$ & $T_{\mathrm{g}, \text { PLLA }}{ }^{a}\left({ }^{\circ} \mathrm{C}\right)$ & $T_{\mathrm{g}, \text { PLLA }}{ }^{b}\left({ }^{\circ} \mathrm{C}\right)$ & $T_{\mathrm{m}, \mathrm{PPDL}}{ }^{c}\left({ }^{\circ} \mathrm{C}\right)$ & $T_{\mathrm{m}, \mathrm{PLLA}}{ }^{c}\left({ }^{\circ} \mathrm{C}\right)$ & $T_{\mathrm{c}}^{d}\left({ }^{\circ} \mathrm{C}\right)$ & $T_{\mathrm{c}, \text { onset }}{ }^{e}\left({ }^{\circ} \mathrm{C}\right)$ \\
\hline P0L100 & 0.00 & 1.00 & - & 52.5 & 53.1 & - & 165.4 & 107.3 & 120.3 \\
\hline P26L74 & 0.27 & 0.73 & -34.3 & 53.0 & 56.0 & 91.7 & 170.7 & 77.9 & 79.8 \\
\hline P52L48 & 0.52 & 0.48 & -32.0 & 44.7 & 49.5 & 92.0 & 163.0 & 77.9 & 79.5 \\
\hline P77L23 & 0.77 & 0.23 & -25.4 & 43.4 & 42.1 & 91.7 & 157.6 & 77.2 & 78.8 \\
\hline P100L0 & 1.00 & 0.00 & -33.2 & - & - & 92.6 & - & 78.7 & 79.8 \\
\hline POL100/P100LO & 0.50 & 0.50 & n.d. & & 54.2 & 90.0 & 169.3 & $73.2 / 101.1$ & $75.4 / 111.2$ \\
\hline
\end{tabular}

${ }^{a} T_{\mathrm{g}}$ taken at the maximum of the loss modulus. ${ }^{b} T_{\mathrm{g}}$ taken at the inflection point. ${ }^{c} T_{\mathrm{m}}$ taken at maximum of melting endotherm. ${ }^{d} T_{\mathrm{c}}$ taken at the maximum of the crystallization exotherm. ${ }^{e} T_{\text {c,onset }}$ taken at the onset of the crystallization exotherm.

end-group (Figure 5b). Two of these distributions can exclusively correspond to copolymers including both PDL and LLA (Figure 5b, blue and orange stars). Again, the cyclic distributions only correspond to both individual homopolymers.

The combination of the results above suggests the occurrence of the following events in the sequential feed reaction of PDL and LLA: Initially PDL is polymerized into PPDL, during which simultaneously rapid inter- and intramolecular transesterification of PPDL occurs resulting in linear and cyclic PPDL chains. ${ }^{9}$ The formed macrocyclic PPDL will also undergo ring-opening again and it is therefore the balance between cyclization reactions and ROP reactions that will yield the equilibrium concentration of the cyclic chains. ${ }^{15}$ After the addition of lactide, the linear PPDL chains initiate the polymerization of LLA forming the poly(PDL-block-LLA) copolymer. From the moment one LLA monomer has been inserted, only LLA monomers can be polymerized or PLLA fragments transesterified. PPDL-blocks of the poly(PDL-blockLLA) copolymers and cyclic PPDL chains remain unaffected. Transesterification of the PLLA block in the poly(PDL-blockLLA) copolymer leads to the formation of cyclic PLLA (which again is the equilibrium resulting from ROP of cyclic PLLA and the cyclization of linear PLLA) and block copolymers having disperse PLLA blocks both consisting of odd and even numbers of lactyl units, while randomization of the poly(PDL-blockLLA) is not observed. Therefore, the final product mixture consists of mainly poly(PDL-block-LLA) copolymer with a $\mathrm{PDO}-$ and an $-\mathrm{H}$ end-group and cyclic homopolymers of PPDL and PLLA as minor components. Each product has a Flory molecular weight distribution as expected for transesterification products.

Thermal Properties and Morphology of Poly(PDLblock-LLA). After it was shown that the sequential feed approach provided an effective strategy to synthesize poly(PDL-block-LLA), this method was used to synthesize a set of 
block copolymers on a somewhat larger scale (15 g) containing different PPDL/PLLA ratios (weight ratios 26/74 (P26L74), 52/48 (P52L48), and 77/23 (P77L23); Table 2). During all sequential feed ROP reactions, both monomers were polymerized to high conversions (>95\%) and number-average molecular weights varying between 60 and $81 \mathrm{~kg} \cdot \mathrm{mol}^{-1}$ were obtained. The reaction mixtures were quenched, washed using methanol and dried, after which DSC, ${ }^{13} \mathrm{C}$ NMR and MALDIToF-MS analyses gave similar results as obtained for the previously prepared poly(PDL-block-LLA) copolymers (PPL1-PPL6). Therefore, P26L74, P52L48, and P77L23 are also assumed to consist of linear poly(PDL-block-LLA) as the major component and cyclic PPDL and PLLA homopolymer as the minor component.

The thermal properties obtained using DSC and DMTA for this set of polymers (including a blend of P0L100/P100LO, weight ratio $=1 / 1$ ) are shown in Table 3 . The $T_{\mathrm{g}}$ of $-33.2^{\circ} \mathrm{C}$ for pure PPDL, taken at the temperature corresponding to the peak of the loss modulus, is in good agreement with literature data, ${ }^{35}$ while the $T_{\mathrm{g}}$ of pure PLLA is around $53{ }^{\circ} \mathrm{C}\left(T_{\mathrm{g}, \infty}=58\right.$ $\left.{ }^{\circ} \mathrm{C}\right){ }^{35}$ Calculated from the $T_{\mathrm{g}}$ values reported by Jamshidi et al., ${ }^{35}$ the observed decrease of the $T_{\mathrm{g}}$ of the PLLA-block with its block length can only partially be attributed to the decrease in molecular weight of the PLLA-block. Furthermore, the $T_{\mathrm{g}}$ of the PPDL-block shows an increase while the $T_{\mathrm{g}}$ of the PLLAblocks decreases, which indicates that the amorphous phases of both blocks are partly miscible. Indeed, calculations for P77L23 using the Fox Equation ${ }^{36}$ lead to a PLLA content of $13 \mathrm{wt} \%$ in the PPDL rich phase, while the PLLA rich phase contains $8 \mathrm{wt}$ $\%$ of PPDL. Most likely, this will consist to a large extend of low molecular weight (cyclic) oligomers, since the solubility of low molecular weight components is generally higher.

The melting and crystallization behavior of the block copolymers shows some interesting features compared to the homopolymers. The PLLA homopolymer shows a melting endotherm at $165.4{ }^{\circ} \mathrm{C}$ and a crystallization exotherm at 120.3 ${ }^{\circ} \mathrm{C}$, while neat PPDL melts at $92.6{ }^{\circ} \mathrm{C}$ and crystallizes at 78.7 ${ }^{\circ} \mathrm{C}$. Even though all block copolymers have two distinct melting peaks, they only show one crystallization peak, which occurs at the same temperature as the crystallization of the PPDL homopolymer. Analysis of the crystallization enthalpy of P26L74 (44.5 J/g) compared to P100L0 (133 J/g) suggests that there is some coincidental crystallization of the PLLA block with the PPDL block. Although less pronounced, this effect is also observable in the other block copolymers. However, this effect can only be considered qualitatively, since it cannot be excluded that the crystallization of PPDL is also impeded by the PLLA block. It is furthermore interesting to note that for all block copolymers used in this study crystallization took place around the crystallization temperature of PPDL, except for PPL5 and PPL6, which consist of 86 and 87 wt \% of PLLA, respectively. The latter block copolymers show an onset of crystallization at $102.3{ }^{\circ} \mathrm{C}$, which relates to the crystallization of PLLA, and a subsequent broad exotherm in which both blocks crystallize. Separate crystallization of the PPDL and PLLA blocks in P26L74 was also observed in DSC when a cooling rate of $2{ }^{\circ} \mathrm{C} \cdot \mathrm{min}^{-1}$ instead of the standard 10 ${ }^{\circ} \mathrm{C} \cdot \mathrm{min}^{-1}$ was used. The influence of the length of the PLLA block on the crystallinity is furthermore reflected in the melting behavior of the PLLA phase. A decrease in melting enthalpy is observed when the block length is decreased, which is larger than the decrease in weight fraction (Supporting Information, Table S3). These results show that the crystallization of PLLA is reduced by an increasing length of the PPDL block, as was also observed for HDPE-PLLA block copolymers. ${ }^{19}$

The morphology of this kind of double crystalline block copolymers is not only dependent on their respective composition, but also on the applied thermal history. ${ }^{32}$ Therefore, in order to obtain both insight into the spherulite growth and the morphology of the submicron domains, the PPDL/PLLA homopolymer blend and all the block copolymers were subjected to both polarized light optical microscopy (PLOM) and transmission electron microscopy (TEM). All samples were subjected to a specific thermal history. Either PLLA was allowed to crystallize first from the melt $\left(200^{\circ} \mathrm{C}\right)$ at temperatures above the melting temperature of PPDL (typically $110-120^{\circ} \mathrm{C}$ ), after which PPDL was crystallized $\left(80{ }^{\circ} \mathrm{C}\right)$, or samples were directly cooled to $80{ }^{\circ} \mathrm{C}$ from the melt, which allowed the simultaneous crystallization of both PPDL and PLLA. When cooled to $120^{\circ} \mathrm{C}$ from the melt, the homopolymer blend shows a spherulitic crystalline growth which stops after $20 \mathrm{~min}$ and is not space filling (Figure 6A).

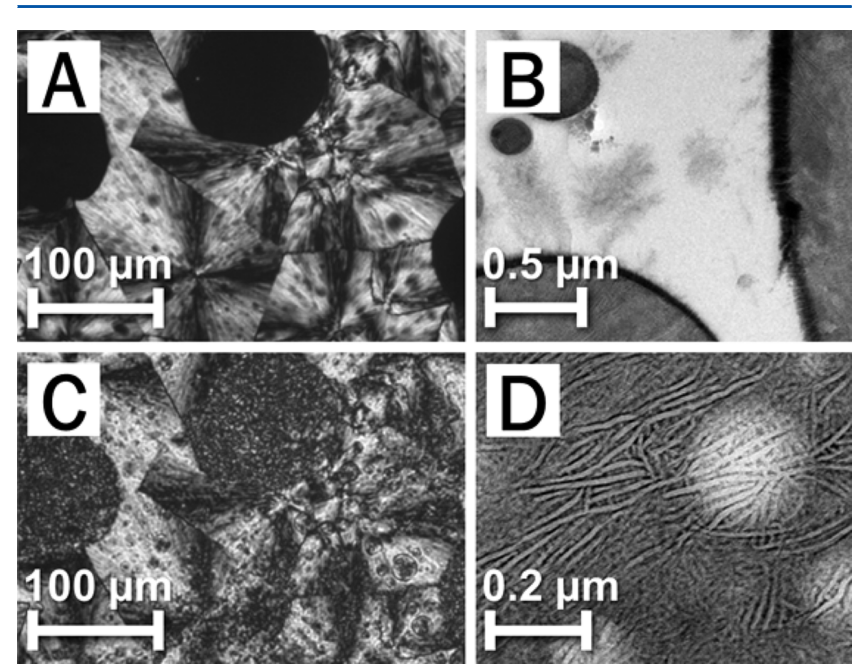

Figure 6. PLOM images of a blend of PPDL and PLLA after $20 \mathrm{~min}$ crystallization from the melt $\left(200{ }^{\circ} \mathrm{C}\right)$ at $120{ }^{\circ} \mathrm{C}(\mathrm{A})$ and subsequent crystallization at $80^{\circ} \mathrm{C}$ for $50 \mathrm{~s}$ (C). TEM images from samples having a similar thermal history (B and D). Dark lamellar domains in TEM correspond to PPDL, light domains to PLLA.

Large noncrystalline domains still remain, as well as smaller domains within the PLLA spherulites. Further cooling to $80^{\circ} \mathrm{C}$ leads to the crystallization of these PPDL domains within seconds, and results in small spherulites due to fast nucleation (Figure 6C). The corresponding TEM analysis shows that besides large PPDL domains, also small domains of PPDL $(<1$ $\mu \mathrm{m}$ ) exist in a PLLA matrix and vice versa (Figure $6 \mathrm{~B}, \mathrm{D})$. The presence of both large domains $(100 \mu \mathrm{m})$ and small domains indicates that in the melt at $200{ }^{\circ} \mathrm{C}$ the system is partially miscible, i.e., there is a PPDL-rich phase containing some PLLA, as well as a PLLA-rich phase containing minor amounts of PPDL. Upon cooling and crystallization the minor component will phase separate further from the major component, as is expected with a system having an upper critical solution temperature (UCST), leading to small dispersed droplets of the minor component. These are the submicrometer domains that are clearly observable in TEM.

The block copolymers prepared on a $15 \mathrm{~g}$ scale were also subjected to a thermal history in which PLLA and PPDL were either allowed to crystallize subsequently or simultaneously 
(see below). For P26L74, crystallization of the PLLA block leads to a well-developed spherulitic morphology, which does not change upon further crystallization of the PPDL block at 80 ${ }^{\circ} \mathrm{C}$ (Figure 7A,B). On the other hand, P52L48 showed a space-

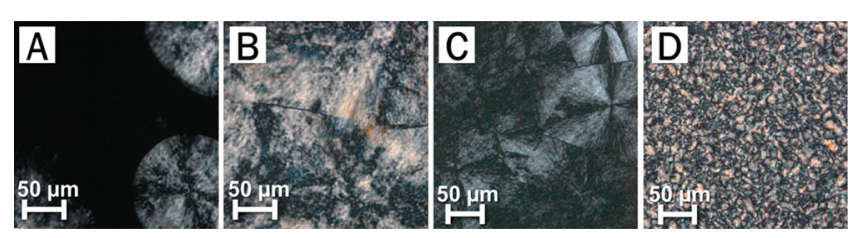

Figure 7. PLOM images of P26L74 after crystallization from the melt $\left(200{ }^{\circ} \mathrm{C}\right.$ ) for $20 \mathrm{~min}$ at $120{ }^{\circ} \mathrm{C}(\mathrm{A}), 50 \mathrm{~min}$ crystallization at $120^{\circ} \mathrm{C}$ (B), and of P52L48 after crystallization from the melt $\left(200^{\circ} \mathrm{C}\right)$ for 20 min at $110{ }^{\circ} \mathrm{C}(\mathrm{C})$ and subsequent crystallization at $80{ }^{\circ} \mathrm{C}$ for $30 \mathrm{~s}$ (D).

filling spherulitic morphology after crystallization at $110{ }^{\circ} \mathrm{C}$, which disappears when the sample is further crystallized at 80 ${ }^{\circ} \mathrm{C}$ (Figure 7C,D), leading to a final morphology similar to samples crystallized directly at $80{ }^{\circ} \mathrm{C}$ from the melt (see Supporting Information for more details). It must be noted that the initial spherulitic morphology for P52L48 is much less intense than the one observed for P26L74, indicating that only a minor part of the PLLA blocks participate in the formation of the spherulites. It was not possible to grow large (observable) PLLA spherulites for P77L23 due to the limited capability of the low molecular weight PLLA block to crystallize. This was also observed in DSC, which, as estimated from the melting enthalpies, shows that the PLLA block in P77L23 has a crystallinity of only $14.5 \%$ relative to the PLLA homopolymer (P0L100). When cooled directly to $80{ }^{\circ} \mathrm{C}$ from the melt, all block copolymers show a similar morphology, having a high number of spherulites per unit area.

Even though the thermal history has a large influence on the crystal growth and morphology, the micromorphology observed with TEM seems to be largely unaffected (Figure 8). P77L23 shows a morphology containing PLLA spheres in a

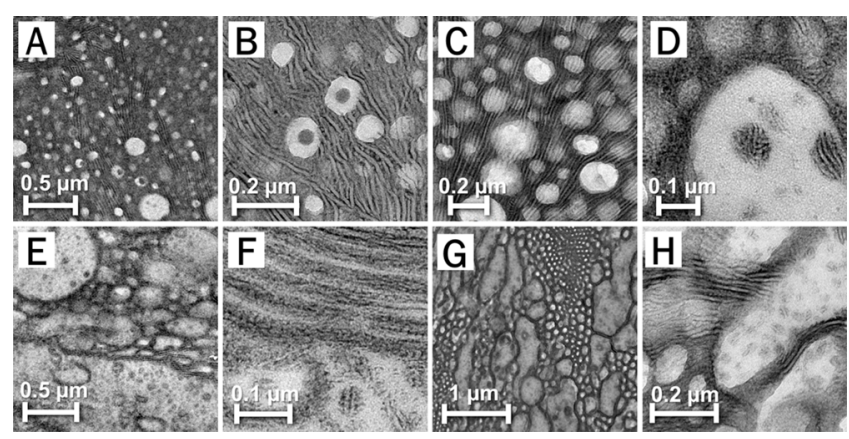

Figure 8. TEM images of P77L23 crystallized from the melt at $80{ }^{\circ} \mathrm{C}$ (A and B) P52L48 crystallized from the melt at $110^{\circ} \mathrm{C}(\mathrm{C}$ and D). P26L74 crystallized from the melt at $80^{\circ} \mathrm{C}(\mathrm{E}$ and F), and crystallized from the melt at $120{ }^{\circ} \mathrm{C}(\mathrm{G}$ and $\mathrm{H})$. Dark lamellar domains in TEM correspond to PPDL, light domains to PLLA.

PPDL matrix, for which the sphere size ranges from 30 to 300 $\mathrm{nm}$ (Figure 8A, B and Supporting Information). This large range of sizes is believed to be caused by the high dispersity of both blocks, leading to the complex situation in which there are many different block copolymer-block lengths present. Short blocks of PLLA can assemble forming small spheres, while the large blocks form the large spheres. Since it was shown that PPDL and PLLA are to some extent miscible, it is not unlikely that very small blocks will not segregate and will actually swell the domain of the large block. Next to this, the cyclic homopolymers that are present will probably also swell both domains. It furthermore should be noted that larger PLLA spheres often contained small PPDL spheres inside as well, which are presumably caused by block copolymers having long PLLA and short PPDL blocks. The PPDL domains are still capable of forming continuous lamellar domains as can clearly be observed in Figure 8B.

Even though P52L48 contains similar amounts of both blocks, it still forms a morphology having dispersed spheres of PLLA and a continuous phase of PPDL, very similar to P77L23 but with larger PLLA domains (Figure 8C,D). Again, PPDL lamellae can be observed in the PLLA domains. Most interestingly, but also most complex, are the morphologies obtained from P26L74, showing spheres of many different sizes, lamellae, and "channels" of PPDL between large PLLA domains which themselves contain smaller PPDL spheres. Again, the complexity of this system is most likely caused by the large dispersity of both blocks, for which it is likely that similar block lengths assemble during the crystallization process forming various domain sizes. It is furthermore remarkable that even though PLLA is the major component, PPDL still forms the continuous phase.

Compatibilization of HDPE/PLLA Blends with Poly(PDL-block-LLA) Copolymers. Compatibilization of a blend using a block copolymer can have several effects on the blend. First, the morphology can change, which in the case of a droplets-in-matrix morphology generally leads to a decrease in droplet size and a homogeneous dispersion. This is the result of a decrease in interfacial tension, leading to more break up of domains in a shear field, and the suppression of coalescence of these domains. ${ }^{37,38}$ Second, the interfacial adhesion between two phases can increase allowing more stress transfer between the phases.

In order to investigate if PPDL is capable of acting as a PE mimic, a set of $12 \mathrm{HDPE} /$ PLLA blends and 12 LLDPE/PLLA blends (see Table 4 for homopolymer properties) were prepared having blend ratios of $80 / 20,50 / 50$, and $20 / 80$ and containing either 0 or 5 wt $\%$ of one of the PPDL-block-PLLA copolymers (see Table 2 ). The blends are named after the wt $\%$ of HDPE or LLDPE compared to PLLA, and the added amount of block copolymer. So, HDPE20 5\% P52L48 is a blend containing a weight ratio of HDPE/PLLA $=20 / 80$ and 5 wt \% of additional P52L48 block copolymer as compatibilizer; i.e., the total blend composition by weight is HDPE/PLLA/ P52L48 19.05/76.19/4.76. The DSC thermograms of the blends show clear melting endotherms for HDPE and PLLA and a crystallization endotherm for HDPE (Figure 9a). Even though slightly obscured by the cold crystallization of PLLA, also a melting endotherm for PPDL can be observed, indicating that the PPDL phase does not cocrystallize with HDPE. This becomes more apparent in the cooling curve, where the crystallization exotherm of PPDL can be identified at $80^{\circ} \mathrm{C}$.

The difference in morphology between noncompatibilized and compatibilized blends can clearly be seen in Figure 10. For HDPE20 and HDPE80, both with and without the addition of block copolymer, a droplets-in-matrix morphology is observed in the scanning electron microscopy (SEM) picture. However, the addition of 5 wt \% of P52L48 drastically reduces the size of the domains and increases the homogeneity of the dispersed 
Table 4. Homopolymers Used in the (Compatibilized) Blends

$\begin{array}{lc}\text { material } & T_{\mathrm{m}}\left({ }^{\circ} \mathrm{C}\right)^{a} \\ \text { HDPE } & 129.2 \\ \text { LLDPE } & 121.6 \\ \text { PLLA } & 173.9\end{array}$

$\mathrm{M}_{\mathrm{n}}\left(\mathrm{kg} \cdot \mathrm{mol}^{-1}\right)$
$12^{c}$
$18^{c}$
$67^{d}$

$\mathrm{M}_{\mathrm{w}}\left(\mathrm{kg} \cdot \mathrm{mol}^{-1}\right)$
$58^{c}$
$96^{c}$
$128^{d}$

$\bigoplus_{\mathrm{M}}$
$4.8^{c}$
$4.4^{c}$
$1.9^{d}$
MFI $\left(\mathrm{g} 10 \mathrm{~min}^{-1}\right)^{b}$
20
2.8
10

${ }^{a}$ Peak melting temperature from DSC. ${ }^{b}$ Taken from datasheet, data at $190{ }^{\circ} \mathrm{C}, 2.16 \mathrm{~kg} .{ }^{c}$ Molecular weight relative to PE standards in TCB at $160{ }^{\circ} \mathrm{C}$. ${ }^{d}$ Molecular weight relative to PS standards in chloroform.

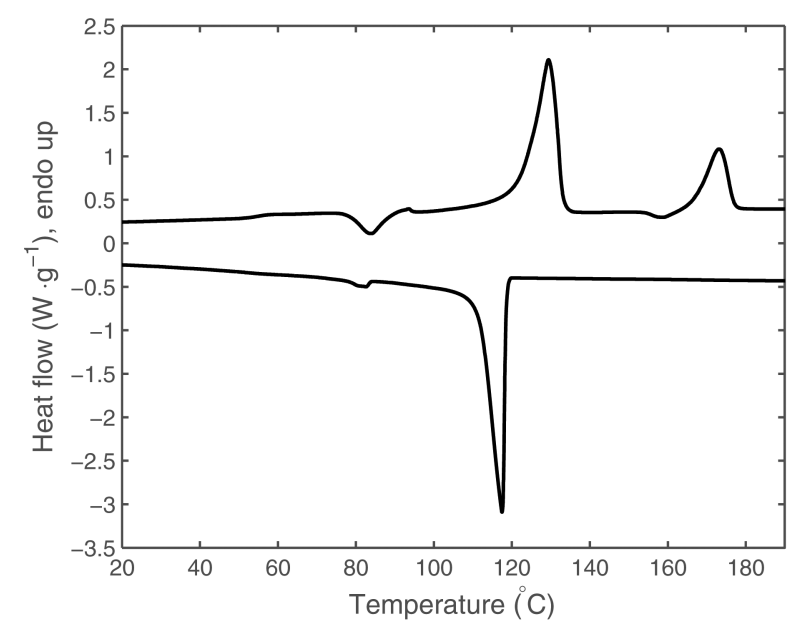

(a)

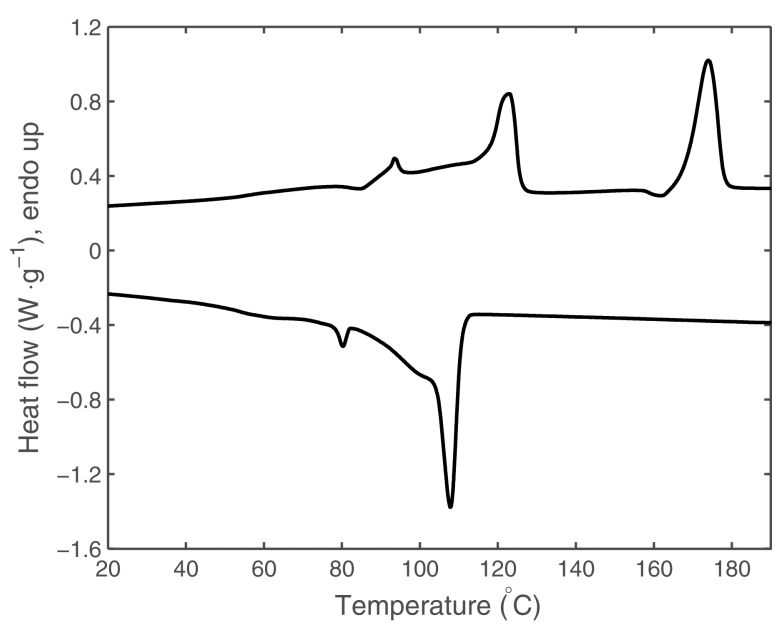

(b)

Figure 9. DSC thermogram of HDPE50 5\% P52L48 (a) and of LLDPE50 5\% P52L48 (b).

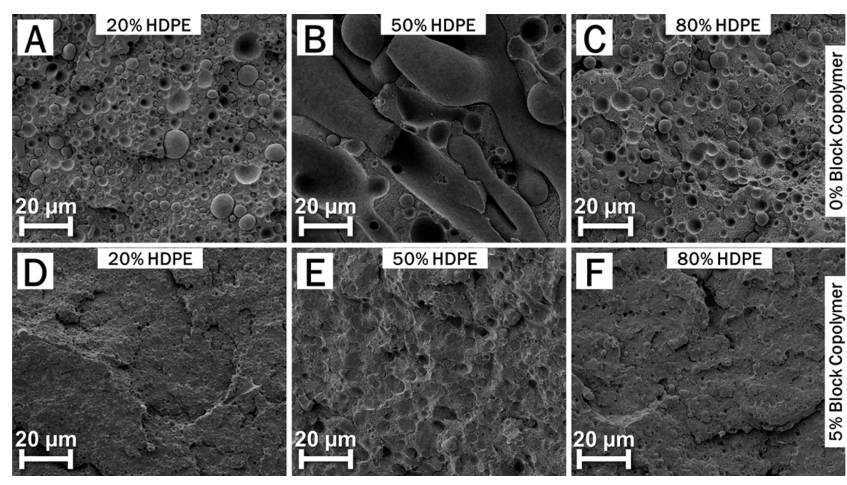

Figure 10. SEM Morphologies of cryogenically fractured PLLA/ HDPE blends containing 0 wt \% $(\mathrm{A}-\mathrm{C})$ and 5 wt \% of P52L48 (DF).

phase. The morphology of neat HDPE50 represents a cocontinuous structure (Figure 10B). In this case, the addition of P52L48 leads to the formation of a droplets-in-matrix morphology. This effect can be explained by considering the apparent cocontinuous phase as a matrix of HDPE with fibers of PLLA, which are formed by the shear forces present during processing. Break up of fibers will lead to the formation of droplets, while coalescence of droplets leads to larger domains which under shear will form fibers again. ${ }^{39}$ The addition of the block copolymer suppresses coalescences of smaller droplets, yielding the droplets-in-matrix structure observed in Figure 10E, which clearly demonstrates the ability of PPDL-blockPLLA copolymers to function as a compatibilizer at the HDPEPLLA interface in the melt.

The cryogenic fracture surfaces investigated using SEM clearly show the effect of the addition of the block copolymers (HDPE20, Figure 11). Without block copolymer (Figure 11A),
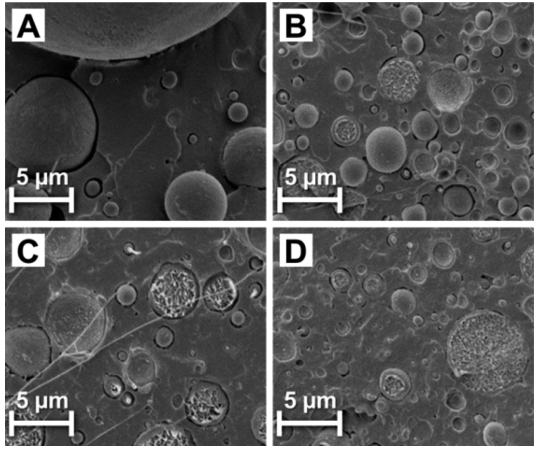

Figure 11. SEM images of cryogenically fractured HDPE20 blends after 10 min quiescent melt-annealing at $200{ }^{\circ} \mathrm{C}$ containing no block copolymer (A), 5 wt \% P26L74 (B), 5 wt \% P52L48 (C), and 5 wt \% P77L23 (D).

a clear void can be observed between the HDPE particles and the PLLA matrix indicating only very little adhesion between the two phases leading to debonding. Furthermore, the dispersed particles show no sign of deformation or fracture. Addition of the block copolymers in all cases leads to a morphology in which the particles are still bonded to the matrix (Figure 11B-D) and clear fracture through the HDPE particles can be observed. This increase in the strength of the interface shows the ability of the PPDL-block-PLLA copolymers to increase the interfacial adhesion, thus functioning as a true compatibilizer for a blend of HDPE and PLLA. Even though the PPDL does not cocrystallize with HDPE, the ability of PPDL to exhibit epitaxial crystallization from the HDPE domains leads to a significant increase in the adhesion. ${ }^{25}$ Therefore, the interfacial morphology is more an interphase, than an interface, in which PPDL functions as a shell layer around the HDPE. To confirm that the block copolymers are 


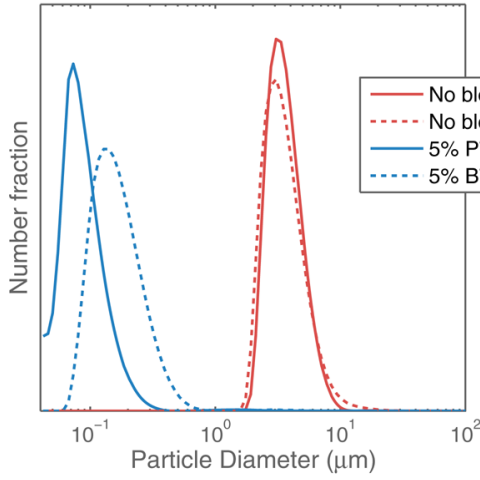

(a)

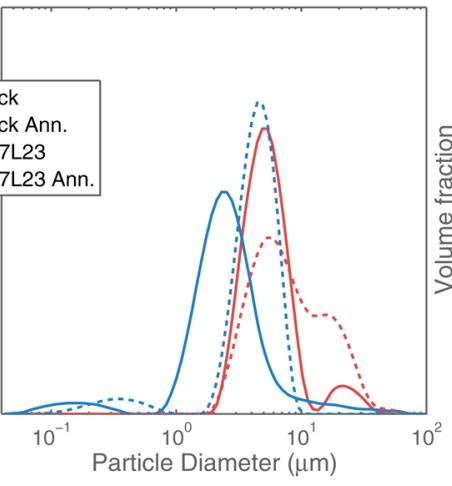

(b)

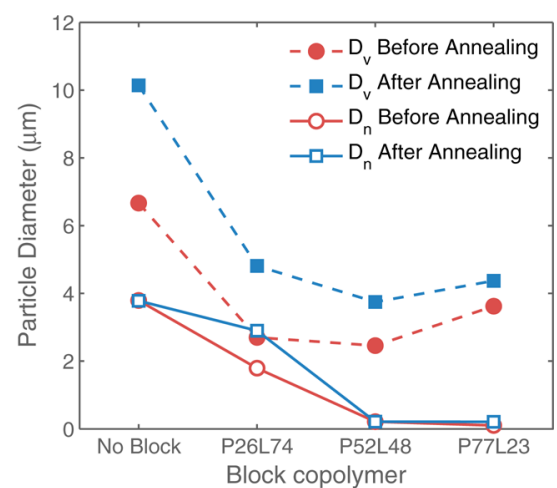

(c)

Figure 12. Number fraction distribution (a) and the volume fraction distribution (b) before and after melt-annealing at $200{ }^{\circ} \mathrm{C}$ for $\mathrm{HDPE20}$ with and without the addition of $5 \mathrm{wt} \%$ P77L23. Number-average particle diameter $\left(D_{n}\right)$ and volume average particle diameter $\left(D_{v}\right)$ before and after melt-annealing vs the type of block copolymer added (c).

responsible for the compatibilization, HDPE20 containing $5 \mathrm{wt}$ $\%$ P100L0 (PPDL homopolymer) was prepared and analyzed, which resulted in a similar morphology as the noncompatibilized HDPE/PLLA blend.

Even though Figure 11 shows a clear difference in interfacial adhesion between noncompatibilized and compatibilized blends, it does not allow quantitative differentiation of the compatibilization efficiency between the different block copolymers (P26L74, P52L48, and P77L23). To obtain more insight into the effect of the relative block lengths on the compatibilization efficiency, the size of the HDPE particles in the HDPE20 blends was investigated by dissolution of the PLLA matrix in chloroform, which does not dissolve HDPE, and subsequent analysis of the obtained dispersions. ${ }^{18}$ The blends containing 5 wt \% of block copolymer show a drastic decrease of the number-average particle size $\left(D_{n}\right)$ from $3.8 \mu \mathrm{m}$ for the noncompatibilized HDPE20 0\% down to $100 \mathrm{~nm}$ for HDPE 5\% P77L23 (Figure 12a). The reason that in SEM the average particle diameter appears larger is the result of a bimodal distribution of particles present in the blends, and is better visualized representing the volume average particle diameter (Figure 12b). The volume average diameter $\left(D_{v}\right)$ for HDPE20 0\% decreases from 6.7 to $3.6 \mu \mathrm{m}$ upon addition of 5 wt \% P77L23, and corresponds to the larger particles observed predominantly in SEM. The presence of the low particle size distribution can be observed in TEM, and was furthermore also observed in DLS measurements (Supporting Information).

To exclude that these particles were block copolymer micelles, the neat block copolymers were measured using both the particle analyzer and DLS in chloroform. Both techniques did not reveal any particle distribution, showing that the block copolymers solubilize in chloroform-confirming that the small particles observed for HDPE20 5\% P52L58/ P77L23 consist of HDPE. Interestingly, decreasing the length of the PPDL block results in an increase of $D_{n}$ to $215 \mathrm{~nm}$ for P52L48 and $1.79 \mu \mathrm{m}$ for P26L74 (Cf. P77L23: $D_{n}=100 \mathrm{~nm}$ ). The steep increase between the latter two is the result of the disappearance of the small particle distribution, for which it appears that a minimal length of the PPDL block is required. Likely, the different distributions are a consequence of the dispersity of both the PPDL and PLLA block $\left(\oplus_{\text {blocks }} \approx 2\right.$ ). Even though P77L23 on average has a three times larger PPDL block compared to the PLLA block, there are also short PPDL blocks with long PLLA blocks present.

Melt-annealing of the blends at $200{ }^{\circ} \mathrm{C}$ was performed in order to examine the efficiency of the block copolymers to suppress coalescence. The neat blend clearly shows coarsening of the domains resulting in a greater amount of large particles. The block copolymer-containing blends show a smaller absolute increase, for which P26L74 appears to be least capable of coalescence suppression, while both P52L48 and P77L23 only show a minor increase in particle size upon meltannealing.

The mechanical properties of the HDPE/PLLA blends were also investigated via tensile testing. However, as a consequence of the intrinsic brittleness of PLLA and the addition of a hard filler (HDPE), analysis of the effect of the block copolymers was impeded. Similar observations were made for PLLA/HDPE systems compatibilized with PLLA-block-HDPE copolymers by Hillmyer et al., who noted that better interfacial adhesion does not necessary lead to better mechanical properties, i.e. an increase in elongation at break or maximum stress, for every system. This was suggested to be related to the inability of HDPE to cavitate in combination with the lack of debonding at the PLLA/HDPE interface. ${ }^{40}$

Compatibilization of LLDPE/PLLA Blends with Poly(PDL-block-LLA) Copolymers. In order to obtain more insight in the compatibilizing efficiency of the block copolymers P26L74, P52L48, and P77L23 and the type polyethylene used, LLDPE/PLLA blends were prepared and investigated. Similar to HDPE, LLDPE50 5\% P52L48 still shows a separate melting and crystallization peak for the PPDL in the block copolymer (Figure 9b), indicating the immiscibility of PPDL and LLDPE at the crystallization temperature. Interestingly, in contradiction to the HDPE blends, the LLDPE20 blends and especially the LLDPE80 blends did not reveal a distinct morphological change in the form of a reduction in particle size using SEM (Supporting Information). Likely this is the result of the higher viscosity of the used LLDPE compared to HDPE, which prevents coalescence of the droplets, effectively reducing the particle size of the noncompatibilized LLDPE blend. This effect becomes clear when the particle sizes obtained from SEM of the noncompatibilized HDPE80 $(\approx 4-6 \mu \mathrm{m})$ are compared to LLDPE80 $(\approx 1-3 \mu \mathrm{m})$. The addition of block copolymer to the LLDPE blends will therefore have a less pronounced effect on the suppression of coalescence, resulting in similar particle 

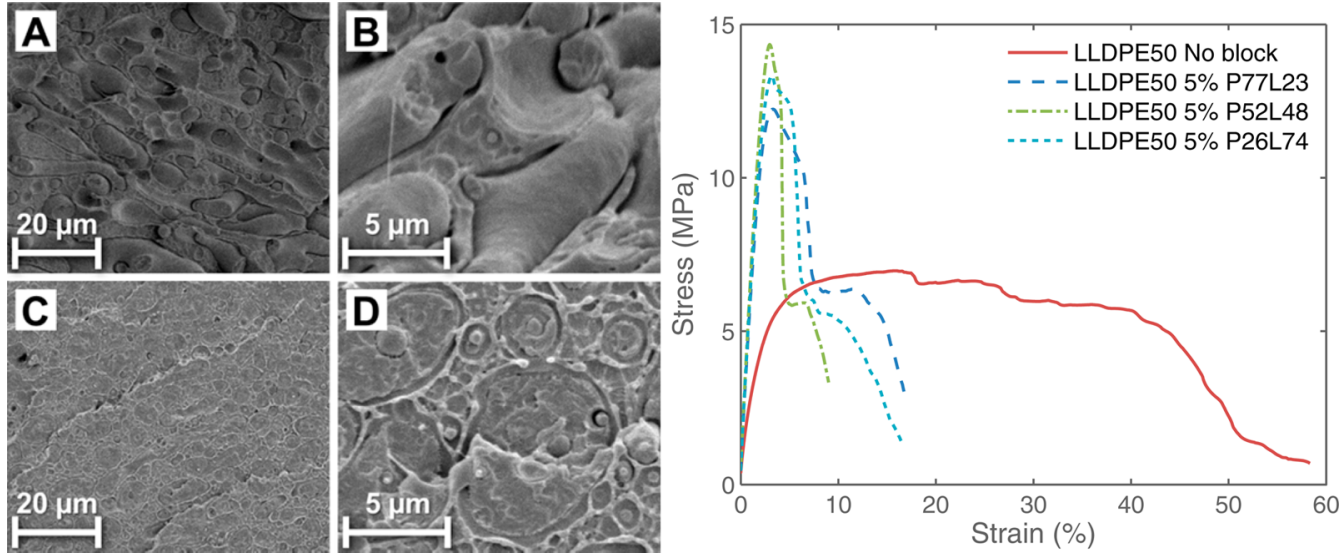

Figure 13. SEM images of LLDPE50 without block copolymer (A, B) and with the addition of 5 wt \% P77L23 (C, D). Stress-strain results for the neat and compatibilized blends (right).

sizes for the compatibilized blends. However, it could still have a significant effect on the interfacial adhesion of the blend.

Conversely, the LLDPE50 blend does show a clear change in morphology upon the addition of block copolymer, shifting from an apparent cocontinuous structure to a droplets-inmatrix morphology, which indicates that PPDL can also function as an LLDPE mimic in the compatibilization of LLDPE/PLLA blends. The change in morphology is reflected in the mechanical behavior of the LLDPE50 blends (Figure 13), which upon the addition of block copolymer show a significantly higher stiffness and strength, while the elongation at break is reduced.

The LLDPE80 blends were also tested for their mechanical behavior. All blends seem to show similar deformation behavior until break at $900 \%$ (Figure 14). However, the uncompatibi-

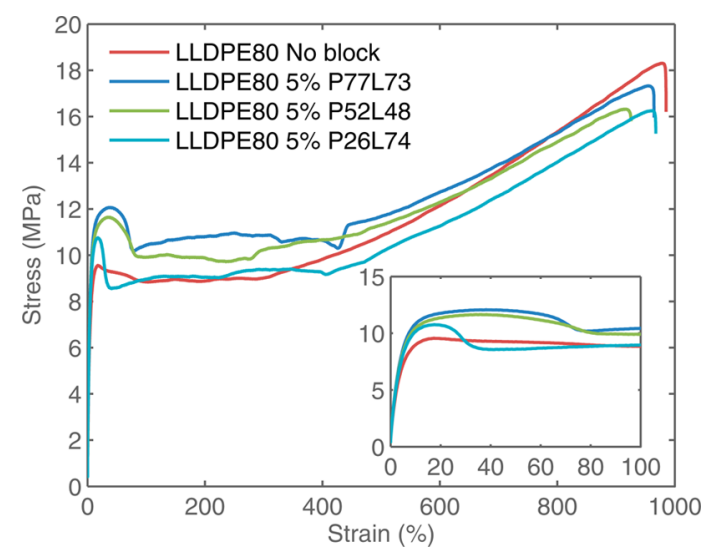

Figure 14. Stress versus strain curves of the tensile test of LLDPE80 containing 0 or 5 wt $\%$ block copolymer.

lized blend LLDPE80 experiences yielding at a significantly lower value compared to the block copolymer-containing blends. PLLA functions as a hard filler, effectively increasing the stress needed to reach the yield point. The morphology of the fracture surfaces of the tensile samples did not reveal deformed PLLA particles. Therefore, it was reasoned that the stress is being transferred between the LLDPE-matrix and the PLLAparticles up to the yield stress, at which debonding and concomitant yielding of the matrix takes place. The addition of block copolymer leads to an increase in interfacial adhesion resulting in a higher yield stress. If there would be no interaction between the LLDPE matrix and the PLLA filler, the yield stress can be expected to reflect $80 \%$ of the value for pure LLDPE. Comparison of this value $\left(\sigma_{\mathrm{LLDPE}, 80 \%}=8.85 \pm 0.23\right.$ $\mathrm{MPa})$ to the obtained yield stresses for neat LLDPE80 $\left(\sigma_{\text {LLDPE80 }}=9.62 \pm 0.23 \mathrm{MPa}\right)$ reveals only a limited increase, resulting from the poor interfacial strength within the noncompatibilized blend. The addition of poly(PDL-blockLLA) does lead to a significant increase of the yield stress $\left(\sigma_{\text {LLDPE80 P26L74 }}=10.8 \pm 0.2 \mathrm{MPa}, \sigma_{\text {LLDPE80 P52L48 }}=11.7 \pm 0.1\right.$ $\mathrm{MPa}, \sigma_{\text {LLDPE80 P77L23 }}=11.9 \pm 0.2 \mathrm{MPa}$ ) as a result of the increased interfacial adhesion. From this it can further be deducted that, similar as for the HDPE blends, P26L74 has less compatibilization efficiency than the block copolymers containing longer PPDL segments, indicating that a certain minimal PPDL block length is required.

The hypothesis that at the yield point debonding occurs was further investigated studying the tensile bars of LLDPE80 containing 5 wt \% of P77L23 and neat LLDPE80 (Figure 15), which were deformed up to $150 \%$. The yielded neck region for both samples shows clear stress whitening, which is presumably the result of an increase in light scattering caused by the formation of voids as a result of debonding. Additionally, noncompatibilized LLDPE80 shows whitening over the reduced width area (Figure 15E), which has not yet yielded, indicating that (partial) debonding of the PLLA particles also occurred in this area. SEM analyses of the cross sections of the regions of interest confirm these statements: the compatibilized blend shows no debonding in the clamp region nor in the nonyielded part of the reduced width area (Figure 15A,B), while the neck area does show regions with debonding (Figure $15 \mathrm{C})$. In contrast, the noncompatibilized blend does show voids as a result of debonding in both the nonyielded and the yielded part of the neck (Figure 15E, F). It should be noted that the morphology for the debonded areas was not homogeneous over the whole cross-section, in which also areas without voids were found.

\section{CONCLUSIONS}

Although aluminum salen catalysts are known to be effective transesterification catalysts, the inability of the salen aluminumlactidyl chain-end to ring-open and insert a PDL monomer as well as its inability of transesterifying a PPDL block was applied to obtain poly(PDL-block-LLA) copolymers by means of a sequential feed copolymerization in which first PDL and subsequently LLA was polymerized. The copolymerization 

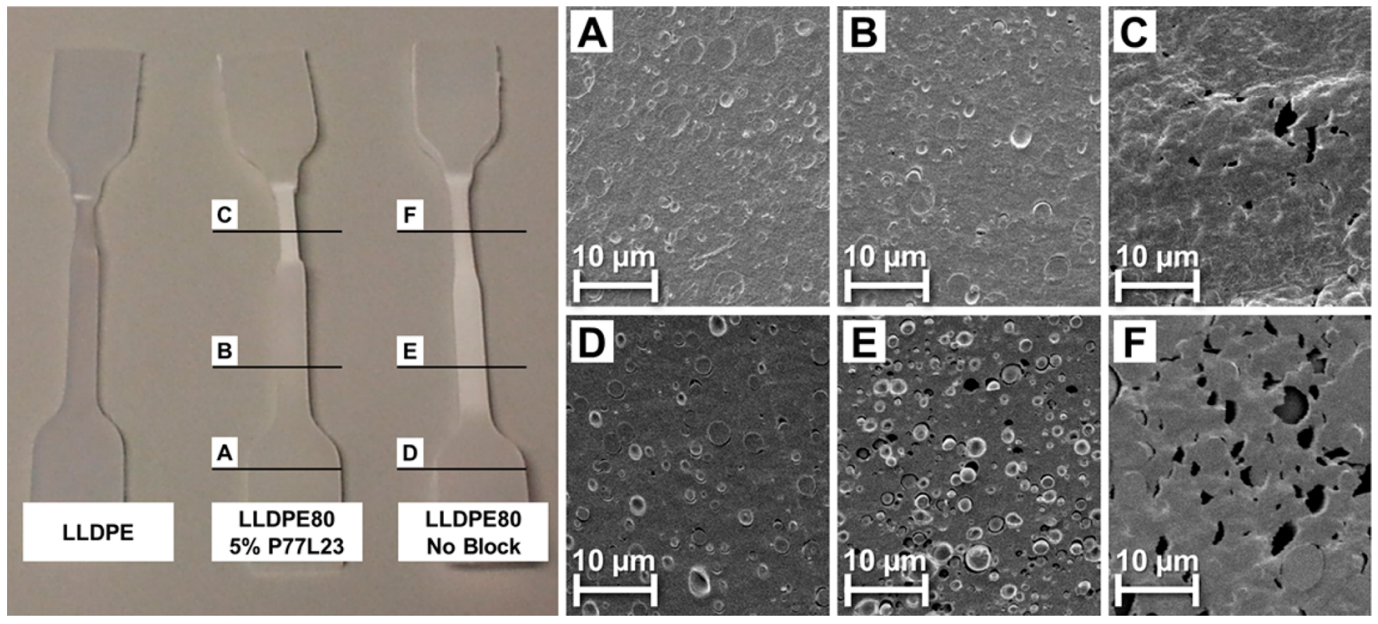

Figure 15. Photo of tensile bars of LLDPE, LLDPE80 containing 5 wt \% P77L23 and neat LLDPE80 showing whitening in the neck region. A-F show the cross sections of the investigated regions of tensile bars as indicated.

afforded an interesting product mixture consisting of poly(PDL-block-LLA) copolymers, in which both blocks have a rather broad dispersity, and cyclic PPDL and PLLA oligomers as a result of transesterification. Curiously, no randomized cyclic or linear poly(PDL-co-LLA) is formed.

The crystallization of the block copolymers suggests coincidental crystallization of both PPDL and PLLA up to a certain block length of the PLLA block. Furthermore, the amorphous phase of both polymers is partially miscible, which shows that the behavior of these block copolymers is in between the block copolymer systems of HDPE-PLLA and HDPE-PCL. ${ }^{32}$ The block copolymers mainly have a dropletsin-matrix morphology in which PPDL is the continuous phase and PLLA comprises the dispersed phase. The high dispersity of the block copolymers results in a wide range of sphere sizes.

Application of the poly(PDL-block-LLA) copolymers as compatibilizers in both HDPE/PLLA and LLDPE/PLLA blends resulted in a clear change of the obtained morphologies for compatibilized blends compared to their noncompatibilized counterparts. For the HDPE-containing blends, this effect was mostly reflected in a significant reduction of particle size, while for the LLDPE-containing blends a strong increase in interfacial adhesion was observed. Furthermore, within the range of block lengths investigated, it was shown that a minimum length for the PPDL block was required for effective compatibilization.

These results show that block copolyesters consisting of a PE-like block and a polar block, which can be produced in a simple cascade process, are considerably more easily synthesized than their PE-based counterparts, especially on large scale. Because of the compatibility of the PE-like PPDL and polyethylenes, poly(PDL-block-LLA) copolymers form an interesting alternative to poly(ethylene-block-LLA) copolymers to be used as compatibilizers for PE/PLLA blends. Moreover, this approach can be extended to other polyethylene/polar polymer blends.

\section{EXPERIMENTAL SECTION}

Reagents and Methods. All solvents and reagents were purchased from commercial sources (Sigma-Aldrich, BioSolve) unless stated otherwise. $p$-Xylene (99.9\%) was dried over sodium and fractionally distilled under nitrogen and degassed prior to use. $\mathrm{PDOH}$ and PDL were freshly distilled from $\mathrm{CaH}_{2}$ under nitrogen prior to use. LLA was kindly received from Purac, recrystallized 3 times from dry toluene and sublimed under vacuum at $95{ }^{\circ} \mathrm{C}$ prior to use. The aluminum Schiff base complexes were synthesized using literature procedure. ${ }^{41}$ All reactions and preparations were either carried out in an MBraun MB-150 GI glovebox or using proper Schlenk techniques. The used HDPE (M200056) and LLDPE (318BE) were kindly provided by Sabic. The PLLA (Synterra 1510) was kindly provided by Synbra.

${ }^{1} \mathrm{H}$ NMR and ${ }^{13} \mathrm{C}$ NMR spectra were recorded in $5 \mathrm{~mm}$ tubes on a Varian Mercury $400 \mathrm{MHz}$ spectrometer equipped with an autosampler at ambient probe temperature in $\mathrm{CDCl}_{3}$. Chemical shifts are reported in ppm vs tetramethylsilane.

Size exclusion chromatography (SEC) was measured on a system equipped with a Shimadzu LC-10ADVP HPLC pump, a Shimadzu RID-10A refractive index detector $\left(35^{\circ} \mathrm{C}\right)$, a Shimadzu SIL-10 ADVP autosampler, a PSS SDV $5 \mathrm{~m}$ guard column followed by 2 PSS SDV linearXL columns in series of $5 \mathrm{~m}(8 \times 300)$ at $35^{\circ} \mathrm{C}$. Chloroform $\left(\mathrm{CHCl}_{3}\right.$, Biosolve) was used as eluent at a flow rate of $1.0 \mathrm{~mL} \cdot \mathrm{min}^{-1}$. The molecular weights were calculated against polystyrene standards (Polymer Laboratories, $M_{\mathrm{p}}=950 \mathrm{~g} \cdot \mathrm{mol}^{-1}$ up to $M_{\mathrm{p}}=2.25 \times 10^{6} \mathrm{~g}$. $\left.\mathrm{mol}^{-1}\right)$. Integration was performed on the part of the distribution containing the linear chains.

High temperature size exclusion chromatography (HT-SEC) of the polyethylenes and PPDL was performed at $160{ }^{\circ} \mathrm{C}$ using a Polymer Laboratories PLXT-20 Rapid GPC polymer analysis system (refractive index detector and viscosity detector) with 3 PLgel Olexis $(300 \times 7.5$ $\mathrm{mm}$, Polymer Laboratories) columns in series. 1,2,4-Trichlorobenzene was used as eluent at a flow rate of $1 \mathrm{~mL} \cdot \mathrm{min}^{-1}$. The molecular weights were calculated with respect to polyethylene standards (Polymer Laboratories). A Polymer Laboratories PL XT-220 robotic sample handling system was used as autosampler.

Differential scanning calorimetry (DSC) analyses of all (co)polymers was carried out on a DSC Q100 from TA Instruments at a heating rate of $10^{\circ} \mathrm{C} \cdot \mathrm{min}^{-1}$. Second runs were recorded after cooling down to ca. $-50{ }^{\circ} \mathrm{C}$. The melting temperatures reported correspond to the melting peaks of the second run.

MALDI-ToF-MS analysis was performed on a Voyager DE-STR from Applied Biosystems equipped with a $337 \mathrm{~nm}$ nitrogen laser. An acceleration voltage of $25 \mathrm{kV}$ was applied. Mass spectra of 1000 shots were accumulated. The polymer samples were dissolved in $\mathrm{CHCl}_{3}$ at a concentration of $1 \mathrm{mg} \cdot 2219 \mathrm{~mL}^{-1}$. The matrix used was trans-2-[3-(4tert-butylphenyl)-2-methyl-2-propenylidene]-malononitrile (DCTB) (Fluka) and was dissolved in chloroform at a concentration of 40 $\mathrm{mg} \cdot \mathrm{mL}^{-1}$. Solutions of matrix, sodium-salt, and polymer were mixed in a volume ratio of $4: 1: 4$, respectively. The mixed solution was handspotted on a stainless steel MALDI target and left to dry. The spectra were recorded in the reflection mode.

Polarized Light Optical microscopy images of crystals were obtained by using a Zeiss LM Axioplan OM, equipped with a Zeiss Axiocam 
camera in polarized transition mode using a linkam heating block for hot-stage examination.

Scanning Electron Microscopy (SEM) micrographs were obtained with a JEOL JSM-5600 scanning electron microscope at an acceleration voltage of $5 \mathrm{kV}$. For the morphology analysis, samples were cryogenically fractured and glued on a SEM stub with conducting carbon paste. For the stress whitening experiments, the tensile bars were cryogenically cut with a razor blade, after which $100-200 \mu \mathrm{m}$ of the surface was trimmed using a Leica Ultracut S/FCS microtome $\left(-130{ }^{\circ} \mathrm{C}\right)$ to obtain the neat surfaces. All samples were then sputter coated with an approximately $10 \mathrm{~nm}$ thick layer of gold using an Emitech K575X sputter coater (90 s, $65 \mathrm{~mA})$.

TEM analysis was done using the following procedure. Before analysis, the samples were trimmed at low $\mathrm{T}\left(-130{ }^{\circ} \mathrm{C}\right)$ and subsequently stained for $20 \mathrm{~h}$ with a $\mathrm{RuO}_{4}$-solution prepared according to Montezinos et al. ${ }^{42}$ Ultrathin sections $(70 \mathrm{~nm})$ were obtained at $-100{ }^{\circ} \mathrm{C}$ using a Leica Ultracut S/FCS microtome. The sections were put on a 200 mesh copper grid with a carbon support layer. The sections were examined in a Tecnai 20 transmission electron microscope, operated at $200 \mathrm{kV}$. PPDL is efficiently stained resulting in a clear distinction between the crystal lamellae (light phase) and intercrystalline amorphous phase (dark domains). Staining has no effect on the PLLA phase, which therefore is light. The lamellae, which sometimes appear to be present in the PLLA domains, e.g. in Figure 6D, are actually lamellae from the PPDL phase, which is on top of the PLLA domain.

Particle size analysis was carried out on a Coulter LS-230 particle analyzer equipped with a small volume module. The apparatus is featured with both a $4 \mathrm{~mW} 750 \mathrm{~nm}$ laser and a Polarization intensity differential scattering (PIDS) tungsten halogen lamp with an output of $150 \mathrm{~lm}$ at $2900 \mathrm{~K}$ to provide a wide measurement range of $0.04-2000$ $\mu \mathrm{m} .300 \mathrm{mg}$ sample was dissolved in $10 \mathrm{~mL}$ chloroform overnight. Each sample was subjected to an ultrasonic treatment of at least 60 min in an ultrasonic bath before analysis to prevent particle coalescence. Three subsequent measurements were recorded to ensure measurement reliability. Presented values for the particle size represent the arithmetical mean value, with the error being equal to the standard deviation.

Further particle diameter analysis was performed on the same samples using dynamic light scattering (DLS), using a Nanotrac Ultra from Microtrac. The laser has a wavelength of $780 \mathrm{~nm}$ and a maximum power of $3 \mathrm{~mW}$. The dispersions were measured with a NPA 250 probe. The dispersions were filtered with a $1 \mu \mathrm{m}$ before measurement.

Tensile tests were performed with a Zwick 100 tensile tester equipped with a $10 \mathrm{kN}$ load cell at room temperature. Tensile test samples were obtained by compression molding the predried blends $\left(60{ }^{\circ} \mathrm{C}\right.$ overnight under vacuum) into plates of $40 \mathrm{~mm} \times 40 \mathrm{~mm} \times 1$ $\mathrm{mm}$ for $10 \mathrm{~min}$ at $200{ }^{\circ} \mathrm{C}$, from which dog bone shaped tensile bars (ISO 527 type B, grip-to-grip separation $=20 \mathrm{~mm}$, gauge length $(\mathrm{LE}=$ $12.5 \mathrm{~mm}$ ) were die cut. The samples were prestressed to $1 \mathrm{~N}$, then loaded until fracture (with the exception of the tensile bars in Figure 15) with a constant cross-head speed of $10 \mathrm{~mm} \cdot \mathrm{min}^{-1}$.

Polymerization Procedure. Before polymerization, a catalyst/ initiator stock solution was prepared by adding an equivalent amount of catalyst and initiator in a vial, dissolving it in $p$-xylene and stirring it for $\pm 12 \mathrm{~h}$ at $100{ }^{\circ} \mathrm{C}$. Formation of the aluminum alkoxides was confirmed by ${ }^{1} \mathrm{H} \mathrm{NMR}$, which showed the complete disappearance of the signals corresponding to the aluminum ethyl protons. In a typical homopolymerization of PDL, PDL $(481 \mathrm{mg}, 2.0 \mathrm{mmol})$ and catalyst stock solution $(0.04 \mathrm{mmol} 2 / \mathrm{PDOH})$ were added to a glass crimp cap vial in a nitrogen filled glovebox and $p$-xylene $(962 \mathrm{mg}$ ) was added after which the solution was distributed over multiple vials. The vials were capped, taken out of the glovebox and placed into a carousel reactor at $100{ }^{\circ} \mathrm{C}$ while stirring with a magnetic stirring bar for $35 \mathrm{~min}$.

For the synthesis of the diblock copolymers, PDL was polymerized as stated above in a crimp cap vial, into which after 35 min of reaction, a hot solution of LLA $(288 \mathrm{mg}, 2.0 \mathrm{mmol})$ in $p$-xylene $(577 \mathrm{mg})$ was syringed, after which the reaction was continued for an additional 10 min. After the reaction the vial was cooled down to room temperature, and a sample was taken to measure the conversion using ${ }^{1} \mathrm{H}$ NMR spectroscopy and to measure the molecular weight distribution using SEC. The reaction mixture was quenched with methanol, washed three times with methanol, and dried under vacuum, which yielded the pure polymers.

For the polymerizations on larger scale the reaction was done as follows. PDL $(8.0 \mathrm{~g}, 33.3 \mathrm{mmol})$, catalyst stock solution $(0.33 \mathrm{mmol}$ $\left.2^{\prime}\right)$ and $p$-xylene $(16.0 \mathrm{~g})$ were added to the glass reactor under inert atmosphere. Subsequently, the reactor temperature was raised to 100 ${ }^{\circ} \mathrm{C}$ and the mixture was allowed to react for $120 \mathrm{~min}$, while being stirred with a mechanical stirrer. Next, a solution of LLA $(8.0 \mathrm{~g}, 55.5$ $\mathrm{mmol})$ in $p$-xylene $(16 \mathrm{~g})$ at $100{ }^{\circ} \mathrm{C}$ was added to the reactor and the reaction was allowed to continue for an additional $60 \mathrm{~min}$. After the reaction was completed, the contents of the reactor were removed and crude samples were taken for ${ }^{1} \mathrm{H}$ NMR spectroscopy and SEC. The reaction mixture was quenched with acidic methanol, washed 3 times with methanol, and dried under vacuum.

Solution Blending. Blends of PPDL and PLLA were prepared by dissolution the desired amount of both polymers in chloroform, after which the solution was precipitated in methanol. The blend was collected by filtration, which was three times washed with methanol, and subsequently dried overnight under vacuum. All samples, including the block copolymers, were subjected to the desired thermal history using a Linkam hot stage under nitrogen flow.

Melt Blend Preparation and Treatment. Before processing, PLLA and the block copolymers were dried overnight in a vacuum oven at $60{ }^{\circ} \mathrm{C}$. Pellets of PLLA, PE and block copolymer powder were subsequently dry-blended in the appropriate amounts. The materials were then melt blended in a laboratory scale corotating mini-twinscrew extruder (Eindhoven University of Technology) with an internal volume of $5 \mathrm{~cm}^{3}$, at $200{ }^{\circ} \mathrm{C}$ under nitrogen flow, with a screw speed of $100 \mathrm{rpm}$. After $10 \mathrm{~min}$, the blends were discharged from the extruder and stored for further analysis. The weight ratios are based on the values without compatibilizer, e.g. HDPE20 contains 20 wt \% HDPE and 80 wt \% PLLA, while HDPE20 5\% P77L23 contains 19.05 wt \% HDPE, 76.19 wt \% PLLA and 4.76 wt \% P77L23. Samples were annealed in the melt by compression molding the dry polymer (blends were dried overnight at $60{ }^{\circ} \mathrm{C}$ under vacuum before compression molding) into plates of $40 \mathrm{~mm} \times 40 \mathrm{~mm} \times 1 \mathrm{~mm}$ for $10 \mathrm{~min}$ at 200 ${ }^{\circ} \mathrm{C}$ for the PE/PLLA blends.

\section{ASSOCIATED CONTENT}

\section{Supporting Information}

The Supporting Information is available free of charge on the ACS Publications website at DOI: 10.1021/acs.macromol.5b01620.

Additional reactions, 2D ${ }^{1} \mathrm{H}-{ }^{13} \mathrm{C}$ HSQC NMR, DSC thermograms, thermal data, tensile tests of PPDL and LDPE homopolymer, DMTA analysis, PLOM, SEM, TEM, particle size analysis, and DLS (PDF)

\section{AUTHOR INFORMATION}

\section{Corresponding Author}

*Telephone: +31 40247 4918. Fax: +31 40246 3966. E-mail: r.duchateau@tue.nl.

\section{Notes}

The authors declare no competing financial interest.

\section{ACKNOWLEDGMENTS}

Financial support by SABIC for this work is gratefully acknowledged.

\section{REFERENCES}

(1) Yanjarappa, M. J.; Sivaram, S. Prog. Polym. Sci. 2002, 27, 13471398.

(2) Chung, T. C. Prog. Polym. Sci. 2002, 27, 39-85. 
(3) Inoue, Y.; Matyjaszewski, K. J. Polym. Sci., Part A: Polym. Chem. 2004, 42, 496-504.

(4) Ouchi, M.; Terashima, T.; Sawamoto, M. Chem. Rev. 2009, 109, 4963-5050.

(5) Wang, Y.; Hillmyer, M. A. Macromolecules 2000, 33, 7395-7403.

(6) Ring, J. O.; Thomann, R.; Mülhaupt, R.; Raquez, J.-M.; Degée, P.; Dubois, P. Macromol. Chem. Phys. 2007, 208, 896-902.

(7) Cai, J.; Liu, C.; Cai, M.; Zhu, J.; Zuo, F.; Hsiao, B. S.; Gross, R. A. Polymer 2010, 51, 1088-1099.

(8) Pepels, M. P. F.; Koeken, R. A. C.; van der Linden, S. J. J.; Heise,

A.; Duchateau, R. Macromolecules 2015, 48, 4779.

(9) Pepels, M. P. F.; Bouyahyi, M.; Heise, A.; Duchateau, R. Macromolecules 2013, 46, 4324-4334.

(10) Bouyahyi, M.; Pepels, M. P. F.; Heise, A.; Duchateau, R. Macromolecules 2012, 45, 3356-3366.

(11) Jasinska-Walc, L.; Hansen, M. R.; Dudenko, D.; Rozanski, A.; Bouyahyi, M.; Wagner, M.; Graf, R.; Duchateau, R. Polym. Chem. 2014, 5, 3306-3310.

(12) Kumar, A.; Kalra, B.; Dekhterman, A.; Gross, R. A. Macromolecules 2000, 33, 6303-6309.

(13) Van der Meulen, I.; Li, Y.; Deumens, R.; Joosten, E. A. J.; Koning, C. E.; Heise, A. Biomacromolecules 2011, 12, 837-843.

(14) Fuoco, T.; Meduri, A.; Lamberti, M.; Venditto, V.; Pellecchia, C.; Pappalardo, D. Polym. Chem. 2015, 6, 1727-1740.

(15) Pepels, M. P. F.; Souljé, P.; Peters, R.; Duchateau, R. Macromolecules 2014, 47, 5542-5550.

(16) Bouyahyi, M.; Duchateau, R. Macromolecules 2014, 47, 517524.

(17) Inkinen, S.; Hakkarainen, M.; Albertsson, A. C.; Södergård, A. Biomacromolecules 2011, 12, 523-532.

(18) Wang, Y.; Hillmyer, M. A. J. Polym. Sci., Part A: Polym. Chem. 2001, 39, 2755.

(19) Müller, A. J.; Castillo, R. V.; Hillmyer, M. A. Macromol. Symp. 2006, 242, 174-181.

(20) Castillo, R. V.; Müller, A. J.; Lin, M.-C.; Chen, H.-L.; Jeng, U.-S.; Hillmyer, M. A. Macromolecules 2008, 41, 6154-6164.

(21) Thurber, C. M.; Xu, Y.; Myers, J. C.; Lodge, T. P.; Macosko, C. W. ACS Macro Lett. 2015, 4, 30-33.

(22) Stempfle, F.; Ritter, B. S.; Mülhaupt, R.; Mecking, S. Green Chem. 2014, 16, 2008-2014.

(23) Pepels, M. P. F.; Hansen, M. R.; Goossens, H.; Duchateau, R. Macromolecules 2013, 46, 7668-7677.

(24) Ortmann, P.; Mecking, S. Macromolecules 2013, 46, 7213-7218.

(25) Pepels, M. P. F.; Kleijnen, R. G.; Goossens, H.; Duchateau, R.; Tandler, R.; Martens, H.; Soliman, M. Unpublished work.

(26) Todd, R.; Tempelaar, S.; Lo Re, G.; Spinella, S.; McCallum, S. A.; Gross, R. A.; Raquez, J.-M.; Dubois, P. ACS Macro Lett. 2015, 4, 408-411.

(27) Spinella, S.; Cai, J.; Samuel, C.; Zhu, J.; McCallum, S. A; Habibi, Y.; Raquez, J.-M.; Dubois, P.; Gross, R. A. Biomacromolecules 2015, 16, $1818-1826$.

(28) Chisholm, M. H.; Choojun, K.; Gallucci, J. C.; Wambua, P. M. Chem. Sci. 2012, 3, 3445-3457.

(29) Balasanthiran, V.; Chisholm, M. H.; Choojun, K.; Durr, C. B. Dalton Trans. 2014, 43, 2781-2788.

(30) Endo, R.; Takeda, M. J. Polym. Sci. 1962, 56, S28-S30.

(31) Schindler, A.; Harper, D. J. Polym. Sci., Polym. Chem. Ed. 1979, 17, 2593-2599.

(32) Castillo, R. V.; Müller, A. J. Prog. Polym. Sci. 2009, 34, 516-560.

(33) Báez, J. E.; Marcos-Fernández, Á.; Galindo-Iranzo, P. J. Polym. Res. 2011, 18, 1137-1146.

(34) Jacobson, H.; Stockmayer, W. H. J. Chem. Phys. 1950, 18, 16001606.

(35) Jamshidi, K.; Ikada, Y. Polymer 1988, 29, 2229-2234.

(36) Fox, T. G. Bull. Am. Phys. Soc. 1956, 1, 123.

(37) Van Puyvelde, P.; Velankar, S.; Moldenaers, P. Curr. Opin. Colloid Interface Sci. 2001, 6, 457-463.

(38) Ajji, A.; Utracki, L. A. Polym. Eng. Sci. 1996, 36, 1574-1585.

(39) Willemse, R. C. Polymer 1999, 40, 2175-2178.
(40) Anderson, K. S.; Hillmyer, M. A. Polymer 2004, 45, 8809-8823. (41) Dzugan, S. J.; Goedken, V. L. Inorg. Chem. 1986, 25, 28582864.

(42) Montezinos, D.; Wells, B. G.; Burns, J. L. J. Polym. Sci., Polym. Lett. Ed. 1985, 23, 421-425. 